\title{
Determination of electroweak parameters in polarised deep-inelastic scattering at HERA
}

\author{
H1 Collaboration and H. Spiesberger (Mainz)
}

V. Andreev ${ }^{19}$, A. Baghdasaryan ${ }^{31}$, K. Begzsuren ${ }^{28}$, A. Belousov ${ }^{19}$, A. Bolz ${ }^{12}$, V. Boudry ${ }^{22}$, G. Brandt ${ }^{40}$, V. Brisson ${ }^{21}$, , D. Britzger ${ }^{12}$, A. Buniatyan ${ }^{2}$, A. Bylinkin ${ }^{42}$, L. Bystritskaya ${ }^{18}$, A. J. Campbel1 ${ }^{10}$, K. B. Cantun Avila ${ }^{17}$, K. Cerny ${ }^{25}$, V. Chekelian ${ }^{20}$, J. G. Contreras ${ }^{17}$, J. Cvach ${ }^{24}$, J. B. Dainton ${ }^{14}$, K. Daum ${ }^{30}$, C. Diaconu ${ }^{16}$, M. Dobre ${ }^{4}$, G. Eckerlin ${ }^{10}$, S. Egli ${ }^{29}$, E. Elsen $^{37}$, L. Favart ${ }^{3}$, A. Fedotov ${ }^{18}$, J. Feltesse ${ }^{9}$, M. Fleischer ${ }^{10}$, A. Fomenko ${ }^{19}$, J. Gayler ${ }^{10}$, L. Goerlich ${ }^{6}$, N. Gogitidze ${ }^{19}$, M. Gouzevitch ${ }^{35}$, C. Grab ${ }^{33}$, A. Grebenyuk ${ }^{3}$, T. Greenshaw ${ }^{14}$, G. Grindhammer ${ }^{20}$, D. Haidt ${ }^{10}$, R. C. W. Henderson ${ }^{13}$, J. Hladkỳ ${ }^{24}$, D. Hoffmann ${ }^{16}$, R. Horisberger ${ }^{29}$, T. Hreus ${ }^{3}$, F. Huber ${ }^{12}$, M. Jacquet ${ }^{21}$, X. Janssen ${ }^{3}$, A. W. Jung ${ }^{43}$, H. Jung ${ }^{10}$, M. Kapichine ${ }^{8}$, J. Katzy ${ }^{10}$, C. Kiesling ${ }^{20}$, M. Klein ${ }^{14}$, C. Kleinwort ${ }^{10}$, R. Kogler ${ }^{11}$, P. Kostka ${ }^{14}$, J. Kretzschmar ${ }^{14}$, D. Krücker ${ }^{10}$, K. Krüger ${ }^{10}$, M. P. J. Landon ${ }^{15}$, W. Lange ${ }^{32}$, P. Laycock ${ }^{14}$, A. Lebedev ${ }^{19}$, S. Levonian ${ }^{10}$, K. Lipka ${ }^{10}$, B. List $^{10}$, J. List ${ }^{10}$, B. Lobodzinski ${ }^{20}$, E. Malinovski ${ }^{19}$, H.-U. Martyn ${ }^{1}$, S. J. Maxfield ${ }^{14}$, A. Mehta ${ }^{14}$, A. B. Meyer ${ }^{10}$, H. Meyer $^{30}$, J. Meyer ${ }^{10}$, S. Mikocki ${ }^{6}$, A. Morozov ${ }^{8}$, K. Müller ${ }^{34}$, Th. Naumann ${ }^{32}$, P. R. Newman ${ }^{2}$, C. Niebuhr ${ }^{10}$, G. Nowak $^{6}$, J. E. Olsson ${ }^{10}$, D. Ozerov ${ }^{29}$, C. Pascaud ${ }^{21}$, G. D. Patel ${ }^{14}$, E. Perez ${ }^{37}$, A. Petrukhin ${ }^{35}$, I. Picuric ${ }^{23}$, D. Pitzl ${ }^{10}$, R. Polifka ${ }^{37}$, V. Radescu ${ }^{44}$, N. Raicevic ${ }^{23}$, T. Ravdandorj ${ }^{28}$, P. Reimer ${ }^{24}$, E. Rizvi $^{15}$, P. Robmann ${ }^{34}$, R. Roosen $^{3}$, A. Rostovtsev ${ }^{41}$, M. Rotaru ${ }^{4}$, D. Šálek ${ }^{25}$, D. P. C. Sankey ${ }^{5}$, M. Sauter ${ }^{12}$, E. Sauvan ${ }^{16,39}$, S. Schmitt $^{10, a}$, L. Schoeffel ${ }^{9}$, A. Schöning ${ }^{12}$, F. Sefkow ${ }^{10}$, S. Shushkevich ${ }^{36}$, Y. Soloviev ${ }^{19}$, P. Sopicki ${ }^{6}$, D. South ${ }^{10}$, V. Spaskov ${ }^{8}$, A. Specka ${ }^{22}$, H. Spiesberger ${ }^{45}$, M. Steder ${ }^{10}$, B. Stella ${ }^{26}$, U. Straumann ${ }^{34}$, T. Sykora ${ }^{3,25}$, P. D. Thompson ${ }^{2}$, D. Traynor ${ }^{15}$, P. Truöl ${ }^{34}$, I. Tsakov ${ }^{27}$, B. Tseepeldorj ${ }^{28,38}$, A. Valkárová ${ }^{25}$, C. Vallée ${ }^{16}$, P. Van Mechelen ${ }^{3}$, Y. Vazdik ${ }^{19, \dagger}$, D. Wegener ${ }^{7}$, E. Wünsch ${ }^{10}$, J. Žáček ${ }^{10}$, Z. Zhang ${ }^{21}$, R. Žlebčík ${ }^{25}$, H. Zohrabyan ${ }^{31}$, F. Zomer ${ }^{21}$

${ }^{1}$ I. Physikalisches Institut der RWTH, Aachen, Germany

${ }^{2}$ School of Physics and Astronomy, University of Birmingham, Birmingham, UK ${ }^{\mathrm{c}}$

${ }^{3}$ Inter-University Institute for High Energies ULB-VUB, Brussels and Universiteit Antwerpen, Antwerp, Belgium ${ }^{\mathrm{d}}$

${ }^{4}$ Horia Hulubei National Institute for R\&D in Physics and Nuclear Engineering (IFIN-HH), Bucharest, Romania ${ }^{j}$

${ }^{5}$ Rutherford Appleton Laboratory, STFC, Didcot, Oxfordshire, UK ${ }^{\mathrm{c}}$

${ }^{6}$ Institute of Nuclear Physics, Polish Academy of Sciences, 31342 Kraków, Poland ${ }^{\mathrm{e}}$

${ }^{7}$ Institut für Physik, TU Dortmund, Dortmund, Germany ${ }^{\mathrm{b}}$

${ }^{8}$ Joint Institute for Nuclear Research, Dubna, Russia

${ }^{9}$ Irfu/SPP, CE Saclay, Gif-sur-Yvette, France

${ }^{10}$ DESY, Hamburg, Germany

${ }^{11}$ Institut für Experimentalphysik, Universität Hamburg, Hamburg, Germany ${ }^{\mathrm{b}}$

12 Physikalisches Institut, Universität Heidelberg, Heidelberg, Germany ${ }^{\mathrm{b}}$

${ }^{13}$ Department of Physics, University of Lancaster, Lancaster, UK $^{\mathrm{c}}$

${ }^{14}$ Department of Physics, University of Liverpool, Liverpool, $\mathrm{UK}^{\mathrm{c}}$

${ }^{15}$ School of Physics and Astronomy, Queen Mary, University of London, London, UK $^{\mathrm{c}}$

${ }^{16}$ Aix Marseille Université, CNRS/IN2P3, CPPM UMR 7346, 13288 Marseille, France

${ }^{17}$ Departamento de Fisica Aplicada, CINVESTAV, Mérida, Yucatán, Mexico ${ }^{\mathrm{h}}$

18 Institute for Theoretical and Experimental Physics, Moscow, Russia ${ }^{\mathrm{i}}$

${ }^{19}$ Lebedev Physical Institute, Moscow, Russia

${ }^{20}$ Max-Planck-Institut für Physik, Munich, Germany

${ }^{21}$ LAL, Université Paris-Sud, CNRS/IN2P3, Orsay, France

${ }^{22}$ LLR, Ecole Polytechnique, CNRS/IN2P3, Palaiseau, France

${ }^{23}$ Faculty of Science, University of Montenegro, Podgorica, Montenegro ${ }^{\mathrm{k}}$

24 Institute of Physics, Academy of Sciences of the Czech Republic, Prague, Czech Republic ${ }^{\mathrm{f}}$

${ }^{25}$ Faculty of Mathematics and Physics, Charles University, Prague, Czech Republic ${ }^{\mathrm{f}}$

${ }^{26}$ Dipartimento di Fisica, Università di Roma Tre and INFN Roma 3, Rome, Italy

${ }^{27}$ Institute for Nuclear Research and Nuclear Energy, Sofia, Bulgaria

${ }^{28}$ Institute of Physics and Technology of the Mongolian Academy of Sciences, Ulaanbaatar, Mongolia

${ }^{29}$ Paul Scherrer Institute, Villigen, Switzerland

${ }^{30}$ Fachbereich C, Universität Wuppertal, Wuppertal, Germany 
31 Yerevan Physics Institute, Yerevan, Armenia

32 DESY, Zeuthen, Germany

${ }^{33}$ Institut für Teilchenphysik, ETH, Zurich, Switzerland ${ }^{\mathrm{g}}$

${ }^{34}$ Physik-Institut der Universität Zürich, Zurich, Switzerland ${ }^{\mathrm{g}}$

35 Université Claude Bernard Lyon 1, CNRS/IN2P3, Villeurbanne, France

${ }^{36}$ Now at Skobeltsyn Institute of Nuclear Physics, Lomonosov Moscow State University, Moscow, Russia

${ }^{37}$ Now at CERN, Geneva, Switzerland

${ }^{38}$ Also at Ulaanbaatar University, Ulaanbaatar, Mongolia

39 Also at LAPP, Université de Savoie, CNRS/IN2P3, Annecy-le-Vieux, France

40 II. Physikalisches Institut, Universität Göttingen, Göttingen, Germany

${ }^{41}$ Now at Institute for Information Transmission Problems RAS, Moscow, Russia ${ }^{1}$

${ }^{42}$ Moscow Institute of Physics and Technology, Dolgoprudny, Moscow Region, Russian Federation ${ }^{m}$

${ }^{43}$ Department of Physics and Astronomy, Purdue University, 525 Northwestern Ave, West Lafayette, IN 47907, USA

${ }^{44}$ Department of Physics, Oxford University, Oxford, UK

${ }^{45}$ PRISMA Cluster of Excellence, Institute of Physics, Johannes Gutenberg-Universität, Mainz, Germany

Received: 5 June 2018 / Accepted: 10 September 2018 / Published online: 26 September 2018

(c) The Author(s) 2018

\begin{abstract}
The parameters of the electroweak theory are determined in a combined electroweak and QCD analysis using all deep-inelastic $e^{+} p$ and $e^{-} p$ neutral current and charged current scattering cross sections published by the $\mathrm{H} 1$ Collaboration, including data with longitudinally polarised lepton beams. Various fits to Standard Model parameters in the on-shell scheme are performed. The mass of the $W$ boson is determined as $m_{W}=80.520 \pm 0.115 \mathrm{GeV}$. The axial-vector and vector couplings of the light quarks to the $Z$ boson are also determined. Both results improve the precision of previous $\mathrm{H} 1$ determinations based on HERA-I data by about a factor of two. Possible scale dependence of the weak coupling parameters in both neutral and charged current interactions beyond the Standard Model is also studied.
\end{abstract}

Dedicated to the memory of our dear friend and colleague Violette Brisson.

a e-mail: sschmitt@mail.desy.de

b Supported by the Bundesministerium für Bildung und Forschung, FRG, under contract numbers 05H09GUF, 05H09VHC, 05H09VHF, 05H16PEA

${ }^{c}$ Supported by the UK Science and Technology Facilities Council, and formerly by the UK Particle Physics and Astronomy Research Council

d Supported by FNRS-FWO-Vlaanderen, IISN-IIKW and IWT and by Interuniversity Attraction Poles Programme, Belgian Science Policy

e Partially Supported by Polish Ministry of Science and Higher Education, Grant DPN/N168/DESY/2009

${ }^{\mathrm{f}}$ Supported by the Ministry of Education of the Czech Republic under the Project INGO-LG14033

g Supported by the Swiss National Science Foundation

${ }^{\text {h }}$ Supported by CONACYT, México, Grant 48778-F

i Russian Foundation for Basic Research (RFBR), Grant no. 1329.2008.2 and Rosatom

${ }^{\mathrm{j}}$ Supported by the Romanian National Authority for Scientific Research under the contract PN 09370101

k Partially Supported by Ministry of Science of Montenegro, no. 05-1/33352

${ }^{1}$ Russian Foundation for Sciences, Project no. 14-50-00150

$\mathrm{m}$ Ministry of Education and Science of Russian Federation Contract no. 02.A03.21.0003

$\dagger$ Deceased
All results are found to be consistent with the Standard Model expectations.

\section{Introduction}

Since the discovery of weak neutral currents in 1973 [1,2], the Glashow-Weinberg-Salam model [3-10] has been established as the theory of electroweak (EW) interactions and as the core of the Standard Model (SM) of particle physics. Already since these early times, deep-inelastic lepton-hadron scattering (DIS) experiments with longitudinally polarised electron beams have provided indispensable results [11,12] for its great success. Nowadays, EW theory has been tested in great detail at lower scales with muon life-time measurements [13] and neutrino scattering experiments [14-18], with precision measurements at the $Z$ pole and at even higher scales [19-24]. The H1 Collaboration has performed first studies of weak interactions at the HERA electron-proton collider in 1993: the measurement of the total chargedcurrent cross section demonstrated for the first time the presence of the $W$-boson propagator [25]. DIS at HERA provides complementary testing ground for studying EW processes at the EW energy scale in the space-like regime. The centre-of-mass energy at HERA nicely fills the gap between low-energy neutrino or muon experiments and highenergy collider experiments, and it offers the possibility to study neutral and charged currents (NC and $\mathrm{CC}$ ) on equal footing.

The H1 experiment [26-29] at the HERA collider recorded collisions of electrons and positrons of $27.6 \mathrm{GeV}$ and unpolarised protons of up to $920 \mathrm{GeV}$ during the HERA-I running period in the years 1992-2000, and the HERA-II running period in the years 2003-2007. These data provide a large set of precise NC and CC cross section measurements. They are an important input to study Quantum Chromodynamics 
(QCD), the theory of the strong force, and are indispensable for exploring the structure of the proton. Furthermore, at the HERA centre-of-mass energy of up to $\sqrt{s}=319 \mathrm{GeV}$, EW effects such as $\gamma Z$ interference significantly contribute to the inclusive NC DIS cross sections at high values of negative four-momentum transfers squared $\left(Q^{2}\right)$. The CC interactions are solely mediated by charged $W$ bosons. This allows for a determination of EW parameters from inclusive $\mathrm{NC}$ and $\mathrm{CC}$ DIS data at high $Q^{2}$ up to $50,000 \mathrm{GeV}^{2}$

At HERA, several determinations of the $W$-boson mass $\left(m_{W}\right)$ have been performed by the $\mathrm{H} 1$ and ZEUS experiments based on different data samples collected during the HERA-I data taking period [30-34]. A first EW analysis was performed using the complete HERA-I data collected by $\mathrm{H} 1$ [35], where the weak neutral-current couplings of the light quarks to the $Z$ boson, the axial-vector $\left(g_{A}^{u / d}\right)$ and vector $\left(g_{V}^{u / d}\right)$ couplings, and $m_{W}$ and the top-quark mass $\left(m_{t}\right)$ were determined. Analyses using $\mathrm{H} 1$ data from HERA-I and HERA-II cross section measurements together with ZEUS data have been reported by the ZEUS Collaboration [36] and by Abt et al. [37].

In the present analysis, the entire set of inclusive NC and CC DIS cross sections measured by the H1 Collaboration during the HERA-I and HERA-II running periods is exploited. The studies thus benefit from the improved statistical precision of the data samples, as compared to the previous analysis [35]. In addition, the longitudinal polarisation of the lepton beams in the HERA-II running provides new sensitivity.

The EW parameters are determined together with the parameters of parton density functions (PDFs) of the proton in combined fits, thus accounting for their correlated uncertainties. The cross section predictions used in this analysis include next-to-next-to-leading order (NNLO) QCD corrections at the hadronic vertex and next-to-leading order (NLO) EW corrections. Within the SM framework the masses of the $W$ and $Z$ bosons and the couplings of the light quarks are determined. Potential modifications from physics beyond the SM are explored. EW parameters are tested in DIS at space-like four-momentum transfer. Therefore, the studies presented here are complementary to measurements of EW parameters at $e^{+} e^{-}$or $p p$ colliders, which are performed in the time-like regime for example at the $Z$ pole or at the $W W$ threshold.

\section{Theoretical framework}

NC interactions in the process $e^{ \pm} p \rightarrow e^{ \pm} X$ are mediated by a virtual photon $(\gamma)$ or $Z$ boson in the $t$-channel, and the cross section is expressed in terms of generalised structure functions $\tilde{F}_{2}^{ \pm}, x \tilde{F}_{3}^{ \pm}$and $\tilde{F}_{\mathrm{L}}^{ \pm}$at EW leading order (LO) as

$$
\begin{aligned}
\frac{d^{2} \sigma^{\mathrm{NC}}\left(e^{ \pm} p\right)}{d x d Q^{2}}= & \frac{2 \pi \alpha^{2}}{x Q^{4}}\left[Y_{+} \tilde{F}_{2}^{ \pm}\left(x, Q^{2}\right)\right. \\
& \left.\mp Y_{-} x \tilde{F}_{3}^{ \pm}\left(x, Q^{2}\right)-y^{2} \tilde{F}_{\mathrm{L}}^{ \pm}\left(x, Q^{2}\right)\right]
\end{aligned}
$$

where $\alpha$ is the fine structure constant and $x$ denotes the Bjorken scaling variable (see e.g. [38]). The helicity dependence of the interaction is contained in the terms $Y_{ \pm}=$ $1 \pm(1-y)^{2}$ with $y$ being the inelasticity of the process. The generalised structure functions can be separated into contributions from pure $\gamma$ - and $Z$-exchange and their interference [39],

$$
\begin{aligned}
\tilde{F}_{2}^{ \pm}= & F_{2}-\left(g_{V}^{e} \pm P_{e} g_{A}^{e}\right) \varkappa_{Z} F_{2}^{\gamma Z} \\
& +\left[\left(g_{V}^{e} g_{V}^{e}+g_{A}^{e} g_{A}^{e}\right) \pm 2 P_{e} g_{V}^{e} g_{A}^{e}\right] \varkappa_{Z}^{2} F_{2}^{Z}, \\
\tilde{F}_{3}^{ \pm}= & -\left(g_{A}^{e} \pm P_{e} g_{V}^{e}\right) \varkappa_{Z} F_{3}^{\gamma Z} \\
& +\left[2 g_{V}^{e} g_{A}^{e} \pm P_{e}\left(g_{V}^{e} g_{V}^{e}+g_{A}^{e} g_{A}^{e}\right)\right] \varkappa_{Z}^{2} F_{3}^{Z},
\end{aligned}
$$

and similarly for $\tilde{F}_{L}$. The variables $g_{A}^{e}$ and $g_{V}^{e}$ stand for the axial-vector and vector couplings of the lepton $e^{ \pm}$to the $Z$ boson, respectively. The degree of longitudinal polarisation of the incoming lepton is denoted as $P_{e}$.

The $Q^{2}$-dependent coefficient $\varkappa_{Z}$ accounts for the $Z$ boson propagator,

$$
\begin{aligned}
\varkappa_{Z}\left(Q^{2}\right) & =\frac{Q^{2}}{Q^{2}+m_{Z}^{2}} \frac{1}{4 \sin ^{2} \theta_{W} \cos ^{2} \theta_{W}} \\
& =\frac{Q^{2}}{Q^{2}+m_{Z}^{2}} \frac{G_{\mathrm{F}} m_{Z}^{2}}{2 \sqrt{2} \pi \alpha} .
\end{aligned}
$$

It can be normalised using the weak mixing angle, $\sin ^{2} \theta_{W}=$ $1-m_{W}^{2} / m_{Z}^{2}$, i.e. using the $W$ and $Z$ boson masses, $m_{W}$ and $m_{Z}$, or the Fermi coupling constant $G_{\mathrm{F}}$, which is measured with high precision in muon-decay experiments [13]. The structure functions are related to linear combinations of the quark and anti-quark momentum distributions, $x q$ and $x \bar{q}$. For instance, the $F_{2}$ and $x F_{3}$ structure functions in the naive quark-parton model, i.e. at LO in QCD, are:

$$
\begin{aligned}
{\left[F_{2}, F_{2}^{\gamma Z}, F_{2}^{Z}\right]=} & x \sum_{q}\left[Q_{q}^{2}, 2 Q_{q} g_{V}^{q}, g_{V}^{q} g_{V}^{q}\right. \\
& \left.+g_{A}^{q} g_{A}^{q}\right]\{q+\bar{q}\}, \\
x\left[F_{3}^{\gamma Z}, F_{3}^{Z}\right]= & x \sum_{q}\left[2 Q_{q} g_{A}^{q}, 2 g_{V}^{q} g_{A}^{q}\right]\{q-\bar{q}\} .
\end{aligned}
$$

The axial-vector and vector couplings of the quarks $q$ to the $Z$ boson, $g_{A}^{q}$ and $g_{V}^{q}$, depend on the electric charge, $Q_{q}$, in units of the positron charge, and on the third component of the weak-isospin of the quarks, $I_{\mathrm{L}, q}^{3}$. In terms of $\sin ^{2} \theta_{W}$, they are given by the standard EW theory:

$g_{A}^{q}=I_{\mathrm{L}, q}^{3}$,

$g_{V}^{q}=I_{\mathrm{L}, q}^{3}-2 Q_{q} \sin ^{2} \theta_{W}$. 
The same formulae also apply to the lepton couplings $g_{A / V}^{e}$.

Universal higher-order corrections, to be discussed below, can be taken into account by introducing $Q^{2}$-dependent form factors $\rho_{\mathrm{NC}, q}$ and $\kappa_{\mathrm{NC}, q}$ [40], replacing Eqs. (7) and (8) by

$g_{A}^{q}=\sqrt{\rho_{\mathrm{NC}, q}} I_{\mathrm{L}, q}^{3}$,

$g_{V}^{q}=\sqrt{\rho_{\mathrm{NC}, q}}\left(I_{\mathrm{L}, q}^{3}-2 Q_{q} \kappa_{\mathrm{NC}, q} \sin ^{2} \theta_{W}\right)$.

The $\mathrm{CC}$ cross section at $\mathrm{LO}$ is written as

$$
\begin{aligned}
& \frac{d^{2} \sigma^{\mathrm{CC}}\left(e^{ \pm} p\right)}{d x d Q^{2}} \\
& =\left(1 \pm P_{e}\right) \frac{G_{\mathrm{F}}^{2}}{4 \pi x}\left[\frac{m_{W}^{2}}{m_{W}^{2}+Q^{2}}\right]^{2}\left(Y_{+} W_{2}^{ \pm}\left(x, Q^{2}\right)\right. \\
& \left.\quad \mp Y_{-} x W_{3}^{ \pm}\left(x, Q^{2}\right)-y^{2} W_{\mathrm{L}}^{ \pm}\left(x, Q^{2}\right)\right) .
\end{aligned}
$$

In the quark-parton model, $W_{\mathrm{L}}^{ \pm}=0$, and the structure functions $W_{2}^{ \pm}$and $x W_{3}^{ \pm}$are obtained from the parton distribution functions. For electron scattering, only positively charged quarks contribute:

$W_{2}^{-}=x(U+\bar{D}), \quad x W_{3}^{-}=x(U-\bar{D})$,

while negatively charged quarks contribute to positron scattering:

$W_{2}^{+}=x(\bar{U}+D), \quad x W_{3}^{+}=x(D-\bar{U})$.

Below the top-quark threshold, one has

$U=u+c, \quad \bar{U}=\bar{u}+\bar{c}, \quad D=d+s, \quad \bar{D}=\bar{d}+\bar{s}$.

Higher-order EW corrections are collected in form factors $\rho_{\mathrm{CC}, e q / e \bar{q}}$. They modify the LO expressions Eqs. (12) and (13) as

$W_{2}^{-}=x\left(\rho_{\mathrm{CC}, e q}^{2} U+\rho_{\mathrm{CC}, e \bar{q}}^{2} \bar{D}\right)$,

$x W_{3}^{-}=x\left(\rho_{\mathrm{CC}, e q}^{2} U-\rho_{\mathrm{CC}, e \bar{q}}^{2} \bar{D}\right)$,

$W_{2}^{+}=x\left(\rho_{\mathrm{CC}, e q}^{2} \bar{U}+\rho_{\mathrm{CC}, e \bar{q}}^{2} D\right)$,

$x W_{3}^{+}=x\left(\rho_{\mathrm{CC}, e \bar{q}}^{2} D-\rho_{\mathrm{CC}, e q}^{2} \bar{U}\right)$.

In the on-shell (OS) scheme [41,42], the independent parameters of the SM EW theory are determined by the fine structure constant $\alpha$ and the masses of the gauge bosons, the Higgs boson $m_{H}$, and the fermions $m_{f}$. The weak mixing angle is then fixed, and $G_{\mathrm{F}}$ is a prediction, given by

$G_{\mathrm{F}}=\frac{\pi \alpha}{\sqrt{2} m_{W}^{2}} \frac{1}{\sin ^{2} \theta_{W}} \frac{1}{(1-\Delta r)}$, where higher-order corrections enter through the quantity $\Delta r=\Delta r\left(\alpha, m_{W}, m_{Z}, m_{H}, m_{t}, \ldots\right)$ [41], which describes corrections to the muon decay beyond the tree-level $[43,44]$.

The $\rho_{\mathrm{NC}}, \kappa_{\mathrm{NC}}$ and $\rho_{\mathrm{CC}}$ parameters are introduced to cover the universal higher-order EW corrections described by loop insertions in the boson propagators. The $\rho_{\mathrm{NC}}$ parameters absorb $Z$-boson propagator corrections combined with higher-order corrections entering the $G_{\mathrm{F}}-m_{W}-\sin ^{2} \theta_{W}$ relation, Eq. (17), while the $\kappa_{\mathrm{NC}}$ parameters absorb one-loop $\gamma Z$ mixing propagator corrections. In addition, there are higherorder corrections to the photon propagator which can be taken into account by using the running fine structure constant. Non-universal corrections due to vertex one-loop Feynman graphs and box diagrams are added separately to the NC cross sections. For the CC cross sections, both universal and nonuniversal corrections can be combined into the form factors $\rho_{\mathrm{CC}, e q / e \bar{q}}$. The dominating corrections in this case are due to loop insertions in the $W$-boson propagator.

One-loop EW corrections have been calculated in Refs. [45-47] for NC and in Refs. [48,49] for CC scattering (see also Ref. [50] for a study of numerical results). The present analysis uses the implementation of EW higher-order corrections in the program EPRC described in Ref. [51]. The size of the purely weak one-loop corrections to the differential cross sections is displayed in Fig. 1 for selected values of $Q^{2}$ for $e^{+} p$ scattering. It includes the $\rho_{\mathrm{NC} / \mathrm{CC}}$ and $\kappa_{\mathrm{NC}}$ form factors, as well as contributions from vertex and box graphs. The corresponding higher order corrections for electron scattering or for non-zero lepton beam polarisation differ by less than 0.01 units from the corrections shown in Fig. 1. Higherorder QED corrections due to real and virtual emission of photons, as well as vacuum polarisation, i.e. the running of the fine structure constant, also have to be taken into account $[52,53]$. These effects, however, had been considered for the cross section measurement and are therefore not included here.

In the OS scheme, used in this analysis, the higher-order correction factors $\rho_{\mathrm{NC}}, \kappa_{\mathrm{NC}}$ and $\rho_{\mathrm{CC}}$ are calculated as a function of $\alpha$ and the input mass values. They depend quadratically on the top-quark mass through $\Delta \rho_{t} \sim m_{t}^{2}$, and logarithmically on the Higgs-boson mass, $\Delta \rho_{H} \sim \ln \left(m_{H}^{2} / m_{W}^{2}\right)$. On the $Z$ pole they amount to about $4 \%$. For DIS at HERA they are of similar size, but they exhibit a nonnegligible $Q^{2}$-dependence [54]. In a modified version of the OS scheme [55], commonly used in QCD analyses of DIS data, the Fermi constant can be used to fix the input parameters replacing the $W$-boson mass as an input parameter. In that case the one-loop corrections are very small, i.e. $\rho_{\mathrm{CC}, e q / e \bar{q}}$ deviate from 1 by a few per mille.

Many extensions of the SM predict modifications of the weak NC couplings. They can be described conveniently by introducing additional parameters $\rho_{\mathrm{NC}}^{\prime}$ and $\kappa_{\mathrm{NC}}^{\prime}$, thus modifying the SM corrections. Also for charged current cross sec- 


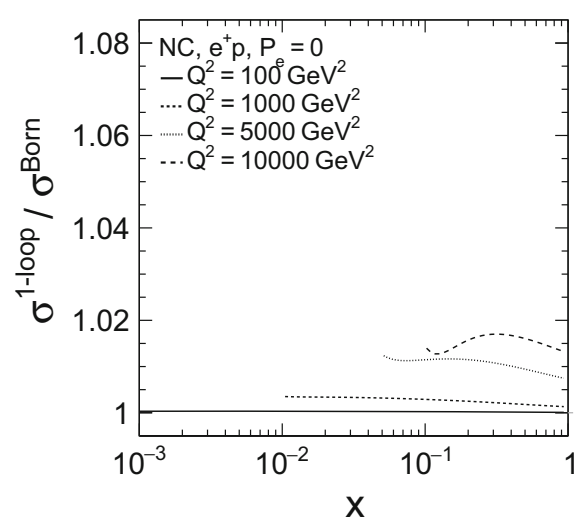

Fig. 1 Size of the purely weak one-loop corrections for the $e^{+} p$ unpolarised inclusive NC DIS (left) and CC DIS (right) cross sections at selected values of $Q^{2}$ as a function of $x$. QED corrections due to real and virtual photons and corrections from the vacuum polarisation (the

tions, similar $\rho_{\mathrm{CC}}^{\prime}$ parameters describing non-standard modifications of the CC couplings can be introduced. The $\rho_{\mathrm{NC}}^{\prime}$, $\kappa_{\mathrm{NC}}^{\prime}$ and $\rho_{\mathrm{CC}}^{\prime}$ are introduced through the following replacements in Eqs. (9), (10), (15) and (16):

$\rho_{\mathrm{NC}} \rightarrow \rho_{\mathrm{NC}}^{\prime} \rho_{\mathrm{NC}}$,
$\kappa_{\mathrm{NC}} \rightarrow \kappa_{\mathrm{NC}}^{\prime} \kappa_{\mathrm{NC}}$,
$\rho_{\mathrm{CC}} \rightarrow \rho_{\mathrm{CC}}^{\prime} \rho_{\mathrm{CC}}$.

In the SM, the parameters $\rho_{\mathrm{NC}}^{\prime}, \kappa_{\mathrm{NC}}^{\prime}$ and $\rho_{\mathrm{CC}}^{\prime}$ are defined to be 1 . Various models with physics beyond the SM predict typical flavour-dependent deviations from 1 and therefore distinct parameters for quarks $\left(\rho_{\mathrm{NC}, q}^{\prime}\right.$ and $\left.\kappa_{\mathrm{NC}, q}^{\prime}\right)$ and for leptons ( $\rho_{\mathrm{NC}, e}^{\prime}$ and $\left.\kappa_{\mathrm{NC}, e}^{\prime}\right)$ are considered. These parameters may also depend on the energy scale. Precision EW measurements on the $Z$ resonance are sensitive to the NC couplings at $m_{Z}$ [19], while DIS is also probing their $Q^{2}$ dependence. For $\mathrm{CC}$ there could be independent modifications $\left(\rho_{\mathrm{CC}}^{\prime}\right)$ for the lepton and quark couplings for each generation. However, only the product of lepton times quark couplings appears in the final expression for the cross section and therefore the same non-standard coupling for all generations is assumed here. Nonetheless, new 4-fermion operators can introduce a difference between electron-quark and electronantiquark scattering, and thus two distinct parameters $\rho_{\mathrm{CC}, e q}^{\prime}$ and $\rho_{\mathrm{CC}, e \bar{q}}^{\prime}$ are considered. These possibly scale-dependent parameters allow for additional tests of the SM couplings.

\section{H1 inclusive DIS cross section data}

This study is based on the entire set of measurements of inclusive NC and CC DIS cross sections by the H1 Collaboration, using data samples for $e^{+} p$ and $e^{-} p$ taken in HERA-I and HERA-II. The measurements are subdivided into two kinematic ranges, corresponding to different sub-

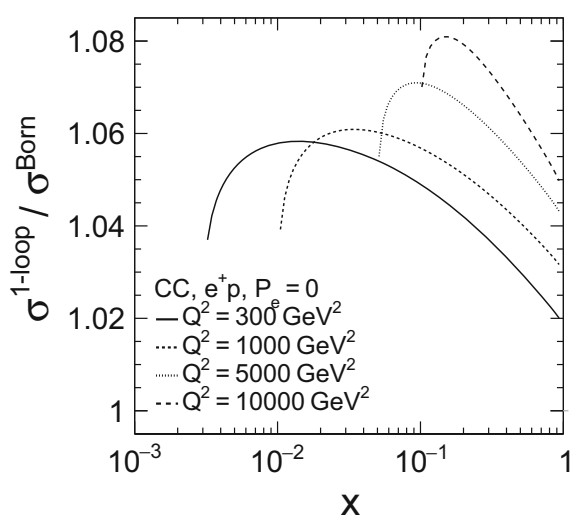

running of $\alpha$ ) are not included. The corrections for electron scattering and for the case of non-vanishing lepton beam polarisation are all very similar to the positron case, such that they differ by less than 0.01 units

detectors where the leptons with small and large scattering angles are identified: low- and medium- $Q^{2}$ for values of $Q^{2}$ typically smaller than $150 \mathrm{GeV}^{2}$ and high- $Q^{2}$ for larger values up to $50,000 \mathrm{GeV}^{2}$. A summary of the data sets used is given in Table 1.

The low- and medium- $Q^{2}$ data sets (data sets 1 and 2) [56] are combined data sets, and they represent all corresponding NC DIS measurements at different beam energies and during different data taking periods published by H1 [56,60-63]. For these data photon exchange dominates over electroweak effects, but they are important in this analysis to constrain the proton PDFs with high precision.

Cross section measurements at high $Q^{2}$ are published separately for the individual data taking periods (data sets: $3-4$ [32], 5-7 [33,57], 8-9 [57], 10-19 [58]). The HERA-II data ${ }^{1}$ were taken with longitudinally polarised lepton beams and exhibit smaller statistical uncertainties due to the increased integrated luminosity, as compared to HERA-I. The high- $Q^{2}$ data provide highest sensitivity for the determination of the EW parameters. The availability of longitudinally polarised lepton beams at HERA-II further improves the sensitivity to the vector couplings $g_{V}^{q}$, as compared to unpolarised data. The data are restricted to $Q^{2} \geq 8.5 \mathrm{GeV}^{2}$, for which quark mass effects are expected to be small, and NNLO QCD predictions $[64,65]$ are expected to provide a good description of the data $[66,67]$.

All the data samples (data sets 1-19) had been corrected for higher-order QED effects due to the emission of photons from the lepton line, photonic lepton vertex corrections, self-energy contributions at the external lepton lines, and fermionic contributions to the running of the fine structure constant (cf. Ref. [32]). QED radiative corrections due to the

\footnotetext{
1 The numerical values of the HERA-II cross sections [58] are corrected to the luminosity measurement erratum [59], by applying the factor 1.018 .
} 
Table 1 Data sets used in the combined EW and QCD fits. For each of the data sets, the corresponding range in $Q^{2}$, the centre-of-mass energy $\sqrt{s}$, the corresponding integrated luminosity values, the number of measured data points, and the average longitudinal polarisation values of the lepton beam are given. During the HERA-I running period data were taken with unpolarised lepton beams. The numbers in brackets denote the respective quantities for the full data set, i.e. without the selection of $Q^{2} \geq 8.5 \mathrm{GeV}^{2}$. The low- and medium- $Q^{2}$ data sets for $\sqrt{s}=319,301,252$ and $225 \mathrm{GeV}$ are combined into two common data sets as described in Ref. [56]. The data sets include electron and positron beams as well as neutral current (NC) and charged current (CC) cross sections. The data sets 10-17 are updated following the discussions in Sect. 3 and in Appendix A

\begin{tabular}{|c|c|c|c|c|c|c|c|}
\hline \multicolumn{2}{|c|}{ Data set } & \multirow{2}{*}{$\frac{Q^{2} \text {-range }\left[\mathrm{GeV}^{2}\right]}{(0.5) 8.5-150}$} & \multirow{2}{*}{$\frac{\sqrt{s}[\mathrm{GeV}]}{301,319}$} & \multirow{2}{*}{$\frac{\mathcal{L}\left[\mathrm{pb}^{-1}\right]}{20,22,97.6}$} & \multirow{2}{*}{$\begin{array}{l}\text { No. of data points } \\
94(262)\end{array}$} & \multirow{2}{*}{$\begin{array}{l}\text { Polarisation [\%] } \\
-\end{array}$} & \multirow{2}{*}{$\begin{array}{l}\text { References } \\
{[56]}\end{array}$} \\
\hline 1 & $e^{+}$combined low- $Q^{2}$ & & & & & & \\
\hline 2 & $e^{+}$combined low- $E_{p}$ & (1.5) 8.5-90 & 225,252 & $12.2,5.9$ & $132(136)$ & - & {$[56]$} \\
\hline 3 & $e^{+} \mathrm{NC} 94-97$ & $150-30,000$ & 301 & 35.6 & 130 & - & {$[32]$} \\
\hline 4 & $e^{+}$CC 94-97 & $300-15,000$ & 301 & 35.6 & 25 & - & {$[32]$} \\
\hline 5 & $e^{-}$NC 98-99 & $150-30,000$ & 319 & 16.4 & 126 & - & [33] \\
\hline 6 & $e^{-}$CC 98-99 & $300-15,000$ & 319 & 16.4 & 28 & - & {$[33]$} \\
\hline 7 & $e^{-}$NC 98-99 high-y & $100-800$ & 319 & 16.4 & 13 & - & [57] \\
\hline 8 & $e^{+}$NC 99-00 & $150-30,000$ & 319 & 65.2 & 147 & - & [57] \\
\hline 9 & $e^{+}$CC 99-00 & $300-15,000$ & 319 & 65.2 & 28 & - & {$[57]$} \\
\hline 10 & $e^{+}$NC L HERA-II & $120-30,000$ & 319 & 80.7 & 136 & $-37.0 \pm 1.0$ & {$[58,59]$} \\
\hline 11 & $e^{+}$CC L HERA-II & $300-15,000$ & 319 & 80.7 & 28 & $-37.0 \pm 1.0$ & {$[58,59]$} \\
\hline 12 & $e^{+}$NC R HERA-II & $120-30,000$ & 319 & 101.3 & 138 & $+32.5 \pm 0.7$ & {$[58,59]$} \\
\hline 13 & $e^{+}$CC R HERA-II & $300-15,000$ & 319 & 101.3 & 29 & $+32.5 \pm 0.7$ & {$[58,59]$} \\
\hline 14 & $e^{-}$NC L HERA-II & $120-50,000$ & 319 & 104.4 & 139 & $-25.8 \pm 0.7$ & {$[58,59]$} \\
\hline 15 & $e^{-}$CC L HERA-II & $300-30,000$ & 319 & 104.4 & 29 & $-25.8 \pm 0.7$ & {$[58,59]$} \\
\hline 16 & $e^{-}$NC R HERA-II & $120-30,000$ & 319 & 47.3 & 138 & $+36.0 \pm 0.7$ & {$[58,59]$} \\
\hline 17 & $e^{-}$CC R HERA-II & $300-15,000$ & 319 & 47.3 & 28 & $+36.0 \pm 0.7$ & {$[58,59]$} \\
\hline 18 & $e^{+}$NC HERA-II high- $y$ & $60-800$ & 319 & 182.0 & 11 & - & {$[58,59]$} \\
\hline 19 & $e^{-}$NC HERA-II high- $y$ & $60-800$ & 319 & 151.7 & 11 & - & {$[58,59]$} \\
\hline
\end{tabular}

exchange of two or more photons between the lepton and the quark lines are small compared to the quoted errors of the QED corrections and had been neglected (cf. Ref. [33]). In the case of CC cross sections, the data had been corrected for $\mathcal{O}(\alpha)$ QED effects at the lepton line (cf. Ref. [32]).

In order to ensure that all first order EW corrections are considered fully and consistently in this analysis, the applied QED corrections to the input data are revisited in detail. In the formulae for the cross section derivation [58], the QED corrections are applied together with acceptance, resolution, and bin-centre corrections, using two independent implementations of the cross section calculations. It turns out that for the HERA-II data (data sets 10-19, Ref. [58]), these two implementations have employed slightly different numerical values for the input EW parameters, and furthermore have considered different components of the higher-order EW corrections. The corrections are therefore re-evaluated and updated values of the previously published cross sections are obtained for this analysis. The procedure is equivalent to the initial cross section determination and therefore does not introduce additional uncertainties. The updated cross sections for the data sets $10-17$, as used in this analysis, are provided in the Appendix A. The differences to the published cross sections are significantly smaller than the statistical uncertainties for any data point. The data sets 18 and 19 are at lower values of $Q^{2}$ and remain unchanged, as well as the HERA-I data (data sets 1-9). The effect of these updates is expected to be small for QCD analyses [58,66,67]. As a cross check, fits similar to H1PDF2012 [58] were performed using either previously published data $[58,59]$ or the corrected data given in the appendix. The two fits are in agreement within experimental uncertainties, where the largest deviations of size one standard deviation are observed for the down-valence contribution at low factorisation scales. In the present analysis the impact is also found to be insignificant, but the updated cross sections are nevertheless applied in order to have best consistency between data and the predictions used in the fits described below.

\section{Methodology}

The EW parameters are determined in fits of the predictions to data, where in addition to the EW parameters of interest also parameters of the PDFs are determined in order to account for PDF uncertainties. The fits are denoted according to their fit 
parameters, for instance ' $m_{W}+$ PDF' denotes a determination of $m_{W}$ together with the parameters of the PDFs.

A dedicated determination of the PDFs in this analysis is important, since all state-of-the-art PDF sets were determined using $\mathrm{H} 1$ data, while assuming that the EW parameters take their SM values. Hence, the use of such PDF sets could bias the results. Furthermore, PDF sets which include the H1 data suffer from the additional complication that the same data were to be used twice, thus leading to underestimated uncertainties.

The parameterisation of the PDFs follows closely the approach of Ref. [66], where the PDF set HERAPDF $2.0^{2}$ was obtained, using EW parameters determined from other experiments. The parameterisation uses five functional forms with altogether 13 fit parameters, defined at the starting scale $Q_{0}^{2}=1.9 \mathrm{GeV}^{2}$. The scale dependence of the PDFs is evaluated using the DGLAP formalism.

As opposed to the HERAPDF2.0 analysis, the ALPOS fitting framework [67] is used in the present analysis. The cross section predictions have been validated against the xFitter framework [58,66,68], which is the successor of the H1Fitter framework [63]. The structure functions are obtained in the zero-mass variable-flavour-number-scheme at NNLO in QCD using the QCDNUM code [69,70]. The one-loop EW corrections are included in an updated version of the EPRC code [51], while the data have already been corrected for higher-order QED radiative effects, as outlined in Sect. 3.

The goodness of fit, $\chi^{2}$, is derived from a likelihood function assuming the quantities to be normal distributed in terms of relative uncertainties $[67,71]$, which is equivalent to lognormal distributed quantities in terms of absolute uncertainties. The log-normal distribution is strictly positive and a good approximation of a Poisson distribution. The latter is important, since in the kinematic domain where the data exhibit the highest sensitivity to the EW parameters, the statistical uncertainties may become sizeable and dominating. The $\chi^{2}$ is calculated as

$$
\chi^{2}=\sum_{i j} \log \frac{\varsigma_{i}}{\tilde{\sigma}_{i}} V_{i j}^{-1} \log \frac{\varsigma_{j}}{\tilde{\sigma}_{j}},
$$

where the sum runs over all data points with measured cross sections $\varsigma_{i}$ and the corresponding theory predictions, $\tilde{\sigma}_{i}$. The covariance matrix $V_{i j}$ is constructed from all relative uncertainties, taking also correlated uncertainties between the data sets into account [58]. The beam polarisation measurements provide four additional data points, included in the vector 5 , with their uncertainties [72] and four corresponding parameters in the fit.

\footnotetext{
${ }^{2}$ HERAPDF2.0 is determined from combined inclusive $\mathrm{NC}$ and $\mathrm{CC}$ data from the $\mathrm{H} 1$ and ZEUS experiments assuming unpolarised lepton beams.
}

The PDF fit alone, i.e. all EW parameters set to their SM values [40], yields a fit quality of $\chi^{2} / n_{\text {dof }}=1432 /(1414-$ $17)=1.03$, where the number of degrees of freedom, $n_{\text {dof }}$, is calculated from 1410 cross section data points plus 4 measurements of the polarisation, and considering $13 \mathrm{PDF}$ and 4 fit polarisation parameters. This indicates an overall good description of the data by the employed model. More detailed studies of the QCD analysis with the given data samples have been presented previously [58,67].

\section{Results}

This section reports the results of different fits, starting with mass determinations in Sect. 5.1, followed by weak NC coupling determinations in Sect. 5.2 and the study of $\rho_{\mathrm{NC}}^{\prime}, \kappa_{\mathrm{NC}}^{\prime}$ and $\rho_{\mathrm{CC}}^{\prime}$ parameters in Sect. 5.3.

\subsection{Mass determinations}

The masses of the $W$ and $Z$ bosons, as well as the top-quark mass are determined using different prescriptions to fix the fit parameters of the EW theory in the OS scheme. The different prescriptions lead to different sensitivities of the measured cross sections to the EW parameters [73]. The results are summarised in Table 2.

In the combined $m_{W}+$ PDF fit, where $\alpha, m_{Z}, m_{t}, m_{H}$ and $m_{f}$ are taken as external input values [40], the EW parameter $m_{W}$ is determined to be

$$
\begin{aligned}
m_{W} & =80.520 \pm 0.070_{\text {stat }} \pm 0.055_{\text {syst }} \pm 0.074_{\mathrm{PDF}} \\
& =80.520 \pm 0.115_{\text {tot }} \mathrm{GeV} .
\end{aligned}
$$

and the expected uncertainty ${ }^{3}$ is $0.118 \mathrm{GeV}$. The total (tot) uncertainty is improved by about a factor of two in comparison to the earlier result based on HERA-I data only [35]. The uncertainty decomposition is derived by switching off the uncertainty sources subsequently or repeating the fit with fixed PDF parameters. ${ }^{4}$ Other uncertainties due to the input masses $\left(m_{Z}, m_{t}, m_{H}\right)$ and theoretical uncertainties, e.g. from incompletely known higher-order terms in $\Delta r$, or model and parameterisation uncertainties of the PDF fit, are all found to be negligible with respect to the experimental uncertainty. The correlation of $m_{W}$ with any of the PDF parameters is weak, with absolute values of the correlation coefficients below 0.2. The global correlation coefficient [75] of $m_{W}$ in the EW+PDF analysis is 0.64 . The $m_{W}$ sensitivity arises predominantly from the $\mathrm{CC}$ data, with the most important constraint being the normalisation through $G_{\mathrm{F}}$ (see Eqs. (11) and

\footnotetext{
3 The expected uncertainty is obtained from a re-fit using the Asimov data set and the data uncertainties [74].

${ }^{4}$ The PDF uncertainty contains both a statistical and a systematic component, but the systematic component dominates.
} 
Table 2 Results for five combined fits of mass parameters together with PDFs. The multiple uncertainties correspond to statistical (stat), experimental systematic (syst) and PDF uncertainties. The $m_{t}$ determination also includes an uncertainty due to the uncertainty of the $W$ mass. The most-right column lists further input parameters not varied in the fit

\begin{tabular}{llc}
\hline Fit parameters & Result & Independent input parameters \\
\hline$m_{W}+\mathrm{PDF}$ & $m_{W}=80.520 \pm 0.070_{\text {stat }} \pm 0.055_{\text {syst }} \pm 0.073_{\mathrm{PDF}} \mathrm{GeV}$ & $\alpha, m_{Z}, m_{t}, m_{H}, m_{f}$ \\
$m_{W}^{\text {prop }}+\mathrm{PDF}$ & $m_{W}^{\text {prop }}=80.62 \pm 0.67_{\text {stat }} \pm 0.17_{\text {syst }} \pm 0.38_{\mathrm{PDF}} \mathrm{GeV}$ & $\alpha, m_{W}, m_{Z}, m_{t}, m_{H}, m_{f}$ \\
$m_{W}^{\left(G_{\mathrm{F}}, m_{W}\right)}+\mathrm{PDF}$ & $m_{W}^{\left(G_{\mathrm{F}}, m_{W}\right)}=82.05 \pm 0.51_{\text {stat }} \pm 0.44_{\text {syst }} \pm 0.37_{\mathrm{PDF}} \mathrm{GeV}$ & $\alpha, G_{\mathrm{F}}, m_{t} m_{H}, m_{f}$ \\
$m_{Z}+\mathrm{PDF}$ & $m_{Z}=91.084 \pm 0.064_{\text {stat }} \pm 0.050_{\text {syst }} \pm 0.070_{\mathrm{PDF}} \mathrm{GeV}$ & $\alpha, m_{W}, m_{t}, m_{H}, m_{f}$ \\
$m_{t}+\mathrm{PDF}$ & $m_{t}=154 \pm 10_{\text {stat }} \pm 12_{\text {syst }} \pm 15_{\mathrm{PDF}} \pm 15_{m_{W}} \mathrm{GeV}$ & $\alpha, m_{W}, m_{Z}, m_{H}, m_{f}$ \\
\hline
\end{tabular}

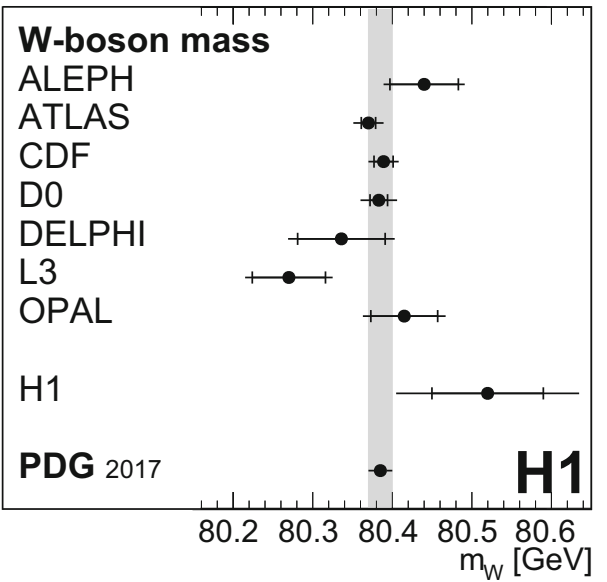

Fig. 2 Value of the $W$-boson mass compared to results obtained by the ATLAS, ALEPH, CDF, D0, DELPHI, L3 and OPAL experiments, and the world average value. The inner error bars indicate statistical uncertainties and the outer error bars full uncertainties

(17)). The highest sensitivity of the $\mathrm{H} 1$ data to $m_{W}$ is at a $Q^{2}$ value of about $3800 \mathrm{GeV}^{2}$. The result for $m_{W}$ is compared to determinations from other single experiments [76-83] in Fig. 2, and is found to be consistent with these as well as with the world average value of $80.385 \pm 0.015 \mathrm{GeV}[40,84]$. The $W$-mass determination in the space-like regime at HERA can be interpreted as an indirect constraint on $G_{\mathrm{F}}$ through Eq. (17), however in a process at large momentum transfer. Using the world average value of $m_{Z}[19,40]$, the result obtained here, $m_{W}=80.520 \pm 0.115 \mathrm{GeV}$, represents an indirect determination of the weak mixing angle in the OS scheme as $\sin ^{2} \theta_{W}=0.22029 \pm 0.00223$. The uncertainty of the present $m_{W}$ determination matches the anticipated HERA results in [73] and in [38,85].

Alternative determinations of $m_{W}$ are also explored. One option is to use exclusively the dependence of the CC cross section on the propagator mass $\sigma^{\mathrm{CC}} \propto\left(m_{W}^{2} /\left(m_{W}^{2}+Q^{2}\right)\right)^{2}$. The result is $m_{W}^{\text {prop }}=80.62 \pm 0.79 \mathrm{GeV}$, with an expected uncertainty of $0.80 \mathrm{GeV}$. This improves the precision of the corresponding fit to HERA-I data [35] by more than a factor of two. The value is consistent with the world average value and with the result of the $m_{W}+\mathrm{PDF}$ fit.
Another $m_{W}$ determination is based on the high precision measurement of $G_{\mathrm{F}}$ [13], which is performed at low energy, together with $\alpha$ as main external input. For this fit, $m_{Z}$ is a prediction and is given by the $G_{\mathrm{F}}-m_{W}-m_{Z}$ relation in Eq. (17). With the precise knowledge of $G_{\mathrm{F}}$, the normalisations of the $\mathrm{CC}$ predictions are known, and therefore the predominant sensitivity to $m_{W}$ arises from the $W$-boson propagator, and the $m_{W}$ dependence through $m_{Z}$ in the NC normalisation is small. In this fit, the value of $m_{W}$, denoted as $m_{W}^{\left(G_{\mathrm{F}}, m_{W}\right)}$, is determined as $m_{W}^{\left(G_{\mathrm{F}}, m_{W}\right)}=82.05 \pm 0.77 \mathrm{GeV}$. The value is consistent at about 2 standard deviations with the world average value and with the result of the $m_{W}+\mathrm{PDF}$ fit above. The larger uncertainty compared to the fit described above is expected. This indirect determination of the $W$-boson mass assumes the validity of the SM [38].

A simultaneous determination of $m_{W}$ and $m_{Z}$ is also performed. The $68 \%$ and $95 \%$ confidence level contours of that $m_{W}+m_{Z}+\mathrm{PDF}$ fit are displayed in Fig. 3 (left). Sizeable uncertainties $\Delta m_{W}=1.4 \mathrm{GeV}$ and $\Delta m_{Z}=1.3 \mathrm{GeV}$ with a very strong correlation are observed. A less strong correlation is found when displaying $\sin ^{2} \theta_{W}=1-m_{W}^{2} / m_{Z}^{2}$ instead of $m_{Z}$ (Fig. 3, right). A mild tension of less than 3 standard deviations between the world average values for $m_{W}$ and $m_{Z}$ and the fit result is observed. The very strong correlation prevents a meaningful simultaneous determination of the two boson masses from the $\mathrm{H} 1$ data alone.

In such a simultaneous determination of two mass parameters, the precise measurement of $G_{\mathrm{F}}$ can be taken as additional input. Due to its great precision it effectively behaves like a constraint, as was proposed earlier $[54,86]$. The $68 \%$ confidence level contours of the $m_{W}+m_{Z}+\mathrm{PDF}$ fit with $G_{\mathrm{F}}$ as one additional input data [13], is further displayed in Fig. 3. As expected, the resulting value of $m_{W}$ is equivalent to the value obtained in the $m_{W}^{\left(G_{\mathrm{F}}, m_{W}\right)}+\mathrm{PDF}$ fit. The $68 \%$ confidence level contour is very shallow due to the high precision of $G_{\mathrm{F}}$. The mild tension with the world average values of $m_{W}$ and $m_{Z}$ is reduced in comparison to the fit without $G_{\mathrm{F}}$ constraint. In the $m_{W}-m_{Z}$ plane the $G_{\mathrm{F}}$ constraint corresponds to a thin band. The orientation of the $m_{W}+m_{Z}+\mathrm{PDF}$ contour is similar to the slope of the $G_{\mathrm{F}}$ band, because the predominant sen- 
Fig. 3 Results of the $m_{W}+m_{Z}+\mathrm{PDF}$ fit, and the $m_{W}+m_{Z}+\mathrm{PDF}$ fit with $G_{\mathrm{F}}$ as additional input. For better visibility, the right panel displays the quantity $\sin ^{2} \theta_{W}=1-m_{W}^{2} / m_{Z}^{2}$ on the vertical axis and identical results as the left panel. The $68 \%$ confidence level (CL) contour of the fit including the $G_{\mathrm{F}}$ measurement is very shallow. The result of the $m_{W}^{\left(G_{\mathrm{F}}, m_{W}\right)}$ fit is further indicated but without uncertainties

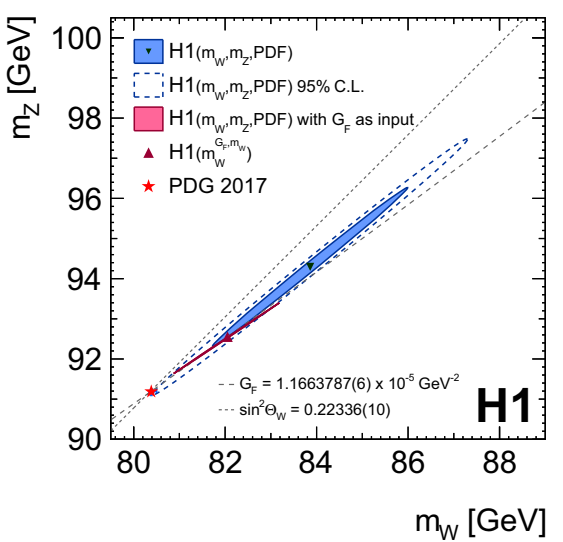

sitivity to $m_{W}$ and $m_{Z}$ of the $\mathrm{H} 1$ data arises through terms proportional to $G_{\mathrm{F}}$ and $\sin ^{2} \theta_{W}$ rather than the propagator terms. This explains the large uncertainty observed in the $m_{W}^{\left(G_{\mathrm{F}}, m_{W}\right)}+\mathrm{PDF}$ fit as compared to the nominal $m_{W}+\mathrm{PDF}$ fit.

The value of $m_{Z}$ is determined in the $m_{Z}+\mathrm{PDF}$ fit to $m_{Z}=$ $91.08 \pm 0.11 \mathrm{GeV}$, to be compared with the measurements at the $Z$ pole of $m_{Z}=91.1876 \pm 0.0021 \mathrm{GeV}$ [19]. The precision is very similar to the $W$-mass determination, as can be expected from Fig. 3 .

The value of $m_{t}$ is determined in the $m_{t}+\mathrm{PDF}$ fit, where $m_{W}$ and $m_{Z}$ are taken as external input, yielding $m_{t}=$ $154 \pm 10_{\text {stat }} \pm 12_{\text {syst }} \pm 15_{\text {PDF }} \pm 15_{m_{W}}$ GeV. The last uncertainty accounts for the $W$-mass uncertainty of $15 \mathrm{MeV}$ [40]. The result is consistent with direct measurements at the LHC [8791] and Tevatron [92]. At HERA, the top quark mass contributes only through loop effects, this explains the moderate sensitivity and the strong dependence on the $W$ mass.

Higher-order corrections to $G_{\mathrm{F}}$ (see Eq. (17), $\Delta r$ ) include bosonic self-energy corrections [55] with a logarithmic dependence on the Higgs-boson mass, $m_{H}$, and thus could, in principle, allow for constraints on $m_{H}$ [73]. At HERA, however, the Higgs-boson mass dependent contribution is too small and no meaningful constraints on $m_{H}$ can be obtained with the HERA data.

A further study on the determination of $\mathrm{EW}$ parameters is performed, by considering the precision measurements of $m_{Z}$ [19], $G_{\mathrm{F}}$ [13], $m_{t}$ [40] and $m_{H}$ [93] as experimental input data in addition to the $\mathrm{H} 1$ data. In this simplified global fit, it is observed that the $\mathrm{H} 1$ data cannot provide significant constraints, for instance on the $W$-boson mass or its correlation to any other parameter. This is because a precision of $7 \mathrm{MeV}$ on $m_{W}$ is already achieved through indirect constraints $[40,94,95]$.

\subsection{Weak neutral-current couplings}

The weak NC couplings, defined in Eqs. (9) and (10), enter the calculation of the structure functions in Eqs. (5) and (6). They are scale dependent beyond the tree-level approxima- tion. The fit parameters for the axial-vector and vector couplings considered here are defined as the tree-level parameters, given in Eqs. (7) and (8). The one-loop corrections are taken into account through multiplicative factors. Results of the fits thus are compared with the SM tree-level predictions for the axial-vector and vector coupling constants. The axialvector and vector couplings of the $u$ - and $d$-type quarks, $g_{A}^{u / d}$ and $g_{V}^{u / d}$, are determined in a combined fit together with the PDF parameters and the results are presented in Table 3 . The two-dimensional contours representing the $68 \%$ confidence level for two fit parameters are displayed and compared ${ }^{5}$ with results from other experiments in Fig. 4 (left). The results are consistent with the SM expectation. The sensitivity on $g_{A}^{u}$ and $g_{V}^{u}$ is similar to LEP and D0 measurements. The HERA measurements do not exhibit sign ambiguities or ambiguities between axial-vector and vector couplings, which are for example present in determinations from $Z$-decays at the pole.

The results for $g_{A}^{u / d}$ and $g_{V}^{u / d}$ obtained from this analysis are found to be compatible with fits, where alternatively external PDFs, such as ABMP16 [97], CT14 [98], H1PDF2017 [67], MMHT14 [99] or NNPDF3.0 [100], are used and the corresponding PDF uncertainties are considered in the $\chi^{2}$ definition. As explained in Sect. 4, this approach yields underestimated uncertainties, but provides a valuable cross check.

By extracting the couplings of the $u$ - and $d$-type quarks separately, i.e. fixing the couplings of the other quark type to their SM expectations and performing a $g_{A}^{u}+g_{V}^{u}+\mathrm{PDF}$ or $g_{A}^{d}+g_{V}^{d}+\mathrm{PDF}$ fit, the uncertainties reduce significantly due to weaker correlations between the fitted quark couplings. The

\footnotetext{
5 It is worth to note that the results are corrected to the Born-level, whereas other experiments often consider effective couplings defined at the $Z$ pole $[19,96]$. Such a fixed-scale definition of couplings is not suitable for DIS, where data cover a wide range of $Q^{2}$ values. On the other hand, the relation between tree-level and effective $Z$-pole couplings is well known (see for example [19]), and the differences of corresponding numerical values are significantly smaller than the achieved precision.
} 
Table 3 Results of the fitted weak neutral-current couplings of the $u$ - and $d$-type quarks. The other parameters $\alpha, m_{W}, m_{Z}$, $m_{t}, m_{H}$ and $m_{f}$ are taken as external input [40]. The uncertainties quoted correspond to the total uncertainties

\begin{tabular}{|c|c|c|c|c|c|}
\hline \multirow[t]{2}{*}{ Fit parameters } & \multirow[t]{2}{*}{ Result } & \multicolumn{4}{|c|}{ Correlations } \\
\hline & & $\overline{g_{A}^{u}}$ & $g_{V}^{u}$ & $g_{A}^{d}$ & $g_{V}^{d}$ \\
\hline \multirow[t]{4}{*}{$g_{A}^{u}+g_{V}^{u}+g_{A}^{d}+g_{V}^{d}+\mathrm{PDF}$} & $g_{A}^{u}=0.614 \pm 0.100$ & 1.00 & & & \\
\hline & $g_{V}^{u}=0.145 \pm 0.056$ & -0.10 & 1.00 & & \\
\hline & $g_{A}^{d}=-0.230 \pm 0.350$ & 0.94 & -0.10 & 1.00 & \\
\hline & $g_{V}^{d}=-0.643 \pm 0.083$ & 0.13 & 0.70 & -0.09 & 1.00 \\
\hline \multirow[t]{2}{*}{$g_{A}^{u}+g_{V}^{u}+\mathrm{PDF}$} & $g_{A}^{u}=0.548 \pm 0.036$ & 1.00 & & & \\
\hline & $g_{V}^{u}=0.270 \pm 0.037$ & -0.18 & 1.00 & & \\
\hline \multirow[t]{2}{*}{$g_{A}^{d}+g_{V}^{d}+\mathrm{PDF}$} & $g_{A}^{d}=-0.619 \pm 0.108$ & & & 1.00 & \\
\hline & $g_{V}^{d}=-0.488 \pm 0.092$ & & & -0.68 & 1.00 \\
\hline
\end{tabular}

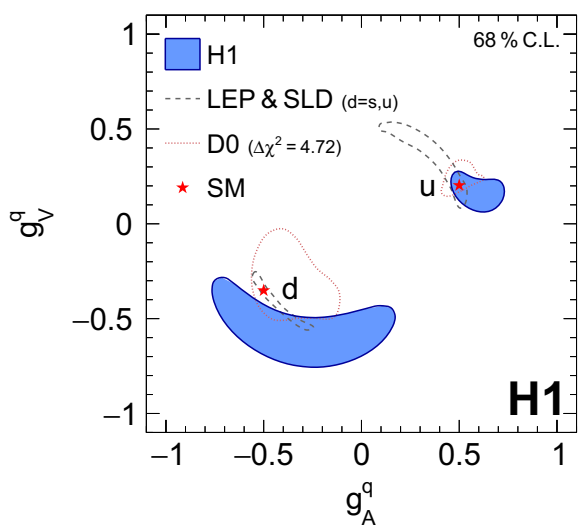

Fig. 4 Results for the weak neutral-current couplings of the $u$ - and $d$-type quarks at the $68 \%$ confidence level (CL) obtained with the $g_{A}^{u}+g_{V}^{u}+g_{A}^{d}+g_{V}^{d}+\mathrm{PDF}$ fit. The left panel shows a comparison with results from the D0, LEP and SLD experiments (the mirror solutions are not shown). The $68 \% \mathrm{CL}$ contours of the $\mathrm{H} 1$ results correspond to

68\% confidence level contours are also displayed in Fig. 4 (right), and numerical values are listed in Table 3 .

\subsection{The $\rho_{\mathrm{NC}}^{\prime}, \kappa_{\mathrm{NC}}^{\prime}$ and $\rho_{\mathrm{CC}}^{\prime}$ parameters}

The values of the $\rho_{\mathrm{NC}, f}^{\prime}$ and $\kappa_{\mathrm{NC}, f}^{\prime}$ parameters (cf. Eqs. (18) and (19)) are determined for $u$ - and $d$-type quarks and for electrons in $\rho_{\mathrm{NC}, u}^{\prime}+\kappa_{\mathrm{NC}, u}^{\prime}+\mathrm{PDF}, \rho_{\mathrm{NC}, d}^{\prime}+\kappa_{\mathrm{NC}, d}^{\prime}+\mathrm{PDF}$ and $\rho_{\mathrm{NC}, e}^{\prime}+\kappa_{\mathrm{NC}, e}^{\prime}+\mathrm{PDF}$ fits, respectively. In these fits, the respective $\rho_{\mathrm{NC}}^{\prime}$ and $\kappa_{\mathrm{NC}}^{\prime}$ parameters are free fit parameters, while the other $\rho^{\prime}$ and $\kappa_{\mathrm{NC}}^{\prime}$ parameters are set to one and the SM EW parameters are fixed. Scale-dependent quantities such as $\rho_{N C, f}, \kappa_{N C, f}, \rho_{C C, f}$ are calculated in the OS scheme as outlined in Sect. 2. The results are presented in Table 4 and the $68 \%$ confidence level contours for the individual light quarks and for electrons are shown in Fig. 5. The results are compatible with the SM expectation at 1-2 standard deviations. The parameters of the $d$-type quarks exhibit larger uncertainties than those of the $u$-type quarks. This is due to the small electric charge of the $d$ quark in the leading $\gamma Z$ interference term (see Eqs. (5) and (6)), and also in $g_{V}^{d}$ (see

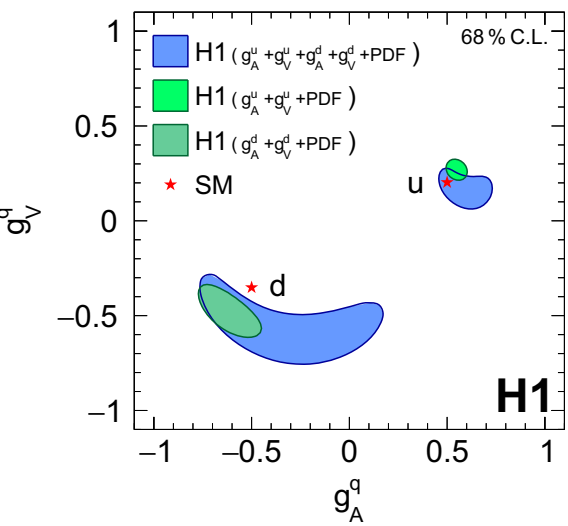

$\Delta \chi^{2}=2.3$, where at the contour all other fit parameters are minimised. The SM expectation is displayed as a star. The right panel shows a comparison of results from fits where the couplings of one quark type are fit parameters, and the couplings of the other quark type are fixed, i.e. the $g_{A}^{u}+g_{V}^{u}+\mathrm{PDF}$ and $g_{A}^{d}+g_{V}^{d}+\mathrm{PDF}$ fits

Eq. (10)). Furthermore, the $d$-valence component of the PDF is smaller than the $u$-valence component.

The results of the $\rho_{\mathrm{NC}, u}^{\prime}+\kappa_{\mathrm{NC}, u}^{\prime}+\mathrm{PDF}$ and $\rho_{\mathrm{NC}, d}^{\prime}+\kappa_{\mathrm{NC}, d}^{\prime}+$ PDF fits (Table 4) are equivalent to the values determined for the NC couplings in $g_{A}^{u}+g_{V}^{u}+\mathrm{PDF}$ and $g_{A}^{d}+g_{V}^{d}+\mathrm{PDF}$ fits, as presented above. The results can be compared to the combined results for $\sin ^{2} \theta_{\text {eff }}^{(u, d)}$ and $\rho_{(u, d)}$ from the LEP+SLD experiments [19]: while the uncertainties are of similar size, the present determinations consider data from a single experiment only.

A simultaneous determination of $\rho_{\mathrm{NC}, u}^{\prime}, \rho_{\mathrm{NC}, d}^{\prime}, \kappa_{\mathrm{NC}, u}^{\prime}$ and $\kappa_{\mathrm{NC}, d}^{\prime}$ is performed, i.e. a $\rho_{\mathrm{NC}, u}^{\prime}+\rho_{\mathrm{NC}, d}^{\prime}+\kappa_{\mathrm{NC}, u}^{\prime}+\kappa_{\mathrm{NC}, d}^{\prime}+\mathrm{PDF}$ fit, and the results are given in the Appendix B. The results are compatible with the SM expectation. These results exhibit sizeable uncertainties, which are due to the very strong correlations between the EW parameters. The exception is $\kappa_{\mathrm{NC}, u}^{\prime}$, which exhibits less strong correlations with the other EW parameters.

Assuming quark universality $\left(\rho_{\mathrm{NC}, q}^{\prime}=\rho_{\mathrm{NC}, u}^{\prime}=\rho_{\mathrm{NC}, d}^{\prime}\right.$ and $\kappa_{\mathrm{NC}, q}^{\prime}=\kappa_{\mathrm{NC}, u}^{\prime}=\kappa_{\mathrm{NC}, d}^{\prime}$, the results of a $\rho_{\mathrm{NC}, q}^{\prime}+\kappa_{\mathrm{NC}, q}^{\prime}+$ PDF fit is presented in Table 4 and displayed in Fig. 5. 
Table 4 Results for $\rho_{\mathrm{NC}}^{\prime}$ and $\kappa_{\mathrm{NC}}^{\prime}$ parameters and their correlation coefficients. The parameters $\alpha, m_{W}, m_{Z}, m_{t}, m_{H}$ and $m_{f}$ are set to their SM values. The uncertainties quoted correspond to the total uncertainties

\begin{tabular}{llll}
\hline Fit parameters & Result & Correlation \\
\hline$\rho_{\mathrm{NC}, u}^{\prime}+\kappa_{\mathrm{NC}, u}^{\prime}+\mathrm{PDF}$ & $\rho_{\mathrm{NC}, u}^{\prime}=1.23 \pm 0.17$ & $\kappa_{\mathrm{NC}, u}^{\prime}=0.88 \pm 0.12$ & 0.61 \\
$\rho_{\mathrm{NC}, d}^{\prime}+\kappa_{\mathrm{NC}, d}^{\prime}+\mathrm{PDF}$ & $\rho_{\mathrm{NC}, d}^{\prime}=1.54 \pm 0.55$ & $\kappa_{\mathrm{NC}, d}^{\prime}=0.74 \pm 0.85$ & 0.92 \\
$\rho_{\mathrm{NC}, e}^{\prime}+\kappa_{\mathrm{NC}, e}^{\prime}+\mathrm{PDF}$ & $\rho_{\mathrm{NC}, e}^{\prime}=1.22 \pm 0.13$ & $\kappa_{\mathrm{NC}, e}^{\prime}=0.98 \pm 0.06$ & 0.74 \\
$\rho_{\mathrm{NC}, d}^{\prime}+\kappa_{\mathrm{NC}, d}^{\prime}+\rho_{\mathrm{NC}, u}^{\prime}+\kappa_{\mathrm{NC}, u}^{\prime}+\mathrm{PDF}$ & $\mathrm{See}$ Appendix B & & \\
$\rho_{\mathrm{NC}, q}^{\prime}+\kappa_{\mathrm{NC}, q}^{\prime}+\mathrm{PDF}$ & $\rho_{\mathrm{NC}, q}^{\prime}=1.20 \pm 0.13$ & $\kappa_{\mathrm{NC}, q}^{\prime}=0.93 \pm 0.11$ & 0.69 \\
$\rho_{\mathrm{NC}, q}^{\prime}+\kappa_{\mathrm{NC}, q}^{\prime}+\rho_{\mathrm{NC}, e}^{\prime}+\kappa_{\mathrm{NC}, e}^{\prime}+\mathrm{PDF}$ & $\mathrm{See}$ Appendix B & & \\
$\rho_{\mathrm{NC}, f}^{\prime}+\kappa_{\mathrm{NC}, f}^{\prime}+\mathrm{PDF}$ & $\rho_{\mathrm{NC}, f}^{\prime}=1.09 \pm 0.07$ & $\kappa_{\mathrm{NC}, f}^{\prime}=0.98 \pm 0.05$ & 0.83 \\
\hline
\end{tabular}

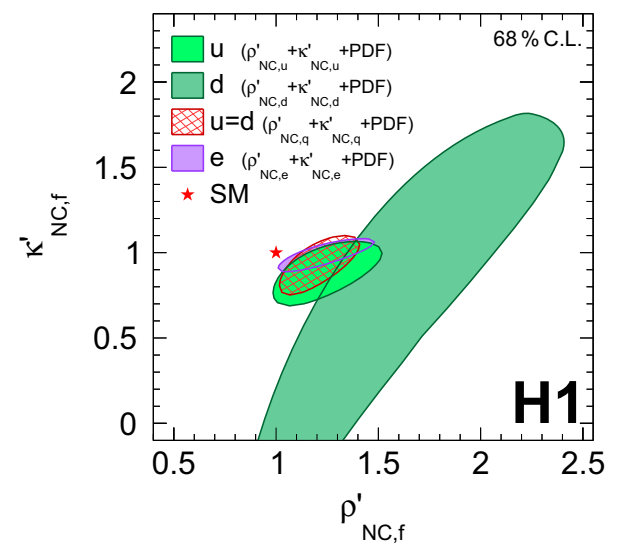

Fig. 5 Results for the $\rho_{\mathrm{NC}, f}^{\prime}$ and $\kappa_{\mathrm{NC}, f}^{\prime}$ parameters for $u$ - and $d$ type quarks and electrons at $68 \%$ confidence level (CL), obtained with the $\rho_{\mathrm{NC}, u}^{\prime}+\kappa_{\mathrm{NC}, u}^{\prime}+\mathrm{PDF}, \rho_{\mathrm{NC}, d}^{\prime}+\kappa_{\mathrm{NC}, d}^{\prime}+\mathrm{PDF}$ and $\rho_{\mathrm{NC}, e}^{\prime}+\kappa_{\mathrm{NC}, e}^{\prime}+\mathrm{PDF}$ fits, respectively. The SM expectation is displayed as a star. The contour of the $d$-type quark is truncated due to the limited scale of the panel. For comparison, also the result of the $\rho_{\mathrm{NC}, q}^{\prime}+\kappa_{\mathrm{NC}, q}^{\prime}+\mathrm{PDF}$ fit is displayed, where quark universality is assumed $(u=d)$. The results of the $\rho_{\mathrm{NC}, u}^{\prime}+\kappa_{\mathrm{NC}, u}^{\prime}+\mathrm{PDF}$ and $\rho_{\mathrm{NC}, d}^{\prime}+\kappa_{\mathrm{NC}, d}^{\prime}+\mathrm{PDF}$ fits are equivalent to the $g_{A}^{u}+g_{V}^{u}+\mathrm{PDF}$ and $g_{A}^{d}+g_{V}^{d}+\mathrm{PDF}$ fits, respectively, displayed in Fig. 4

These determinations are dominated by the $u$-type quark couplings. The $\rho_{\mathrm{NC}, q}^{\prime}$ and $\kappa_{\mathrm{NC}, q}^{\prime}$ parameters can be determined together with the electron parameters $\rho_{\mathrm{NC}, e}^{\prime}$ and $\kappa_{\mathrm{NC}, e}^{\prime}$ in a $\rho_{\mathrm{NC}, q}^{\prime}+\kappa_{\mathrm{NC}, q}^{\prime}+\rho_{\mathrm{NC}, e}^{\prime}+\kappa_{\mathrm{NC}, e}^{\prime}+\mathrm{PDF}$ fit. Results are given in the Appendix B and no significant deviation from the SM expectation is observed.

Assuming the parameters $\rho_{\mathrm{NC}}^{\prime}$ and $\kappa_{\mathrm{NC}}^{\prime}$ to be identical for quarks and leptons, then denoted as $\rho_{\mathrm{NC}, f}^{\prime}$ and $\kappa_{\mathrm{NC}, f}^{\prime}$, these parameters are determined in a $\rho_{\mathrm{NC}, f}^{\prime}+\kappa_{\mathrm{NC}, f}^{\prime}+\mathrm{PDF}$ fit and results are again listed in Table 4 . The values exhibit the smallest uncertainties and no significant deviation from unity is observed as expected in the SM.

The values of the $\rho_{\mathrm{CC}, e q}^{\prime}$ and $\rho_{\mathrm{CC}, e \bar{q}}^{\prime}$ parameters of the $\mathrm{CC}$ cross sections are determined in a $\rho_{\mathrm{CC}, e q}^{\prime}+\rho_{\mathrm{CC}, e \bar{q}}^{\prime}+\mathrm{PDF}$ fit and results are listed in Table 5. The $68 \%$ confidence level contours are shown in Fig. 6. The parameters are found to be consistent with the SM expectation.

Setting the two parameters equal, i.e. $\rho_{\mathrm{CC}, f}^{\prime}=\rho_{\mathrm{CC}, e q}^{\prime}=$ $\rho_{\mathrm{CC}, e \bar{q}}^{\prime}$, a higher precision is achieved. The parameter $\rho_{\mathrm{CC}, f}^{\prime}$ is determined together with the NC parameters in a $\rho_{\mathrm{NC}, f}^{\prime}+\kappa_{\mathrm{NC}, f}^{\prime}+\rho_{\mathrm{CC}, f}^{\prime}+\mathrm{PDF}$ fit to $\rho_{\mathrm{CC}, f}^{\prime}=1.004 \pm 0.008$. The full result of that fit is listed in Appendix B and all values are found to be consistent with the SM expectations. The CC parameter has an uncertainty of $0.8 \%$ and is only weakly correlated with the NC parameters. This indicates that the CC and $\mathrm{NC}$ parameters can be tested independently of each other. The NC parameters are very similar to the ones obtained in the $\rho_{\mathrm{NC}, f}^{\prime}+\kappa_{\mathrm{NC}, f}^{\prime}+\mathrm{PDF}$ fit, as presented in Table 4.

The inclusive NC and $\mathrm{CC}$ cross sections have been measured over a wide range of $Q^{2}$ values at HERA. This can be exploited to perform tests of models beyond the SM where scale-dependent modifications of coupling parameters are predicted. Such tests could not be performed by the LEP and SLD experiments [40].

In order to study the scale dependence of possible extensions of EW parameters in the NC sector the values of $\kappa_{\mathrm{NC}}^{\prime}$ and $\rho_{\mathrm{NC}}^{\prime}$ are determined at different values of $Q^{2}$. The data at $Q^{2} \geq 500 \mathrm{GeV}^{2}$ are subdivided into four $Q^{2}$ ranges and individual $\rho_{\mathrm{NC}}^{\prime}$ and $\kappa_{\mathrm{NC}}^{\prime}$ parameters are assigned to each interval. For $Q^{2} \leq 500 \mathrm{GeV}^{2}$ the SM expectation $\rho_{\mathrm{NC}}^{\prime}=1$ and $\kappa_{\mathrm{NC}}^{\prime}=1$ is used, because of the limited HERA sensitivity to EW effects at low energy scales. All parameters are determined together with a common set of PDF parameters.
Table 5 Results for $\rho_{\text {CC }}^{\prime}$ parameters. The other parameters $\alpha, m_{W}, m_{Z}, m_{t}, m_{H}$ and $m_{f}$ are fixed to their SM values. The uncertainties quoted correspond to the total uncertainties

\begin{tabular}{llll}
\hline Fit parameters & Result & Correlation \\
\hline$\rho_{\mathrm{CC}, e q}^{\prime}+\rho_{\mathrm{CC}, e \bar{q}}^{\prime}+\mathrm{PDF}$ & $\rho_{\mathrm{CC}, e q}^{\prime}=0.983 \pm 0.010$ & $\rho_{\mathrm{CC}, e \bar{q}}^{\prime}=1.088 \pm 0.031$ & -0.50 \\
$\rho_{\mathrm{NC}, f}^{\prime}+\kappa_{\mathrm{NC}, f}^{\prime}+\rho_{\mathrm{CC}, f}^{\prime}+\mathrm{PDF}$ & See Appendix B & & \\
\hline
\end{tabular}




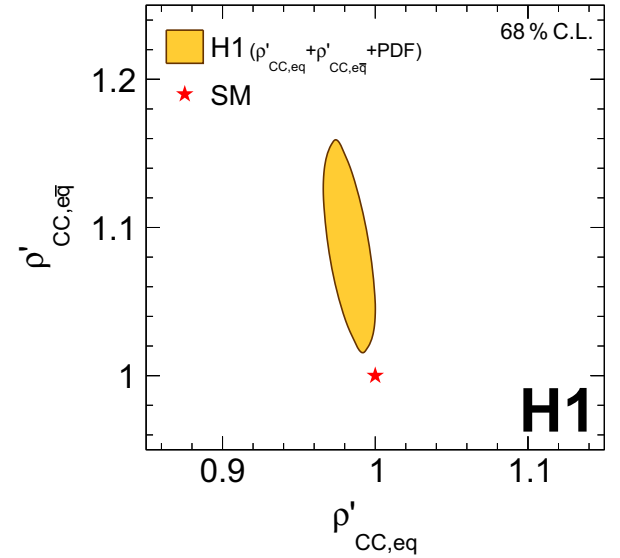

Fig. 6 Results for the $\rho_{\mathrm{CC}, e q}^{\prime}$ and $\rho_{\mathrm{CC}, e \bar{q}}^{\prime}$ parameters at the $68 \%$ confidence level (CL) obtained with the $\rho_{\mathrm{CC}, e q}^{\prime}+\rho_{\mathrm{CC}, e \bar{q}}^{\prime}+\mathrm{PDF}$ fit

Three separate fits are performed: first, for determining in each $Q^{2}$ range two quark parameters $\rho_{\mathrm{NC}, q}^{\prime}$ and $\kappa_{\mathrm{NC}, q}^{\prime}$ assuming $\rho_{\mathrm{NC}, q}^{\prime}=\rho_{\mathrm{NC}, u}^{\prime}=\rho_{\mathrm{NC}, d}^{\prime}$ and $\kappa_{\mathrm{NC}, q}^{\prime}=\kappa_{\mathrm{NC}, u}^{\prime}=\kappa_{\mathrm{NC}, d}^{\prime}$, while setting the lepton parameters to unity; second, for determining the lepton parameters $\kappa_{\mathrm{NC}, e}^{\prime}$ and $\rho_{\mathrm{NC}, e}^{\prime}$ while setting the quark parameters to unity; third, for determining fermion parameters $\kappa_{\mathrm{NC}, f}^{\prime}$ and $\rho_{\mathrm{NC}, f}^{\prime}$ common to both quarks and the lepton assuming $\rho_{\mathrm{NC}, f}^{\prime}=\rho_{\mathrm{NC}, u}^{\prime}=\rho_{\mathrm{NC}, d}^{\prime}=\rho_{\mathrm{NC}, e}^{\prime}$ and $\kappa_{\mathrm{NC}, f}^{\prime}=\kappa_{\mathrm{NC}, u}^{\prime}=\kappa_{\mathrm{NC}, d}^{\prime}=\kappa_{\mathrm{NC}, e}^{\prime}$. Results for the $\rho_{\mathrm{NC}}^{\prime}$ and $\kappa_{\mathrm{NC}}^{\prime}$ parameters are presented in Fig. 7 and are given in Appendix B. The values of $\rho_{\mathrm{NC}}^{\prime}$ and $\kappa_{\mathrm{NC}}^{\prime}$ in different $Q^{2}$ intervals are largely uncorrelated, while the two parameters $\rho_{\mathrm{NC}}^{\prime}$ and $\kappa_{\mathrm{NC}}^{\prime}$ within any given $Q^{2}$ interval have strong correlations. The highest sensitivity to the $\kappa_{\mathrm{NC} f}^{\prime}$ parameter of about $6 \%$ is found at about $\sqrt{Q^{2}} \sim 60 \mathrm{GeV}$. The results are found to be consistent with the SM expectation and no significant scale dependence is observed.

The possible scale dependence of the CC couplings is studied by determining the $\rho_{\mathrm{CC}}^{\prime}$ parameters for different values of $Q^{2}$. A total of three fits are performed, where either $\rho_{\mathrm{CC}, e q}^{\prime}$ or $\rho_{\mathrm{CC}, e \bar{q}}^{\prime}$ (cf. Eq. (20)) or $\rho_{\mathrm{CC}, f}^{\prime}$ is scale dependent. The CC data are grouped into four $Q^{2}$ intervals. Results of the $\rho_{\mathrm{CC}}^{\prime}$ parameters are presented in Fig. 8 and are given in the Appendix B. The parameters $\rho_{\mathrm{CC}, e \bar{q}}^{\prime}$ have uncertainties of about $4 \%$ over a large range in $Q^{2}$, and the parameters $\rho_{\mathrm{CC}, e q}^{\prime}$ are determined with a precision of $1.3 \%$ to $3 \%$ over the entire kinematically accessible range. The $\rho_{\mathrm{CC}, f}^{\prime}$ parameters are determined with high precision of $1.0 \%$ to $1.8 \%$ over the entire $Q^{2}$ range. The values are found to be consistent with the SM expectation of unity. These studies represent the first determination of the $\rho_{\mathrm{CC}}^{\prime}$ parameters for separate quark flavours and also its first scale dependence test.

The studies on the scale dependence of the $\rho^{\prime}$ and $\kappa^{\prime}$ parameters provide tests of the SM formalism. Investigations of specific models beyond the Standard Model such as contact interactions or leptoquarks, also using the full $\mathrm{H} 1$ data sample, have been published previously [101,102].

\section{Summary}

Parameters of the electroweak theory are determined from all neutral current and charged current deep-inelastic scattering cross section measurements published by H1, using NNLO QCD and one-loop electroweak predictions. The inclusion of the cross section data from HERA-II with polarised lepton

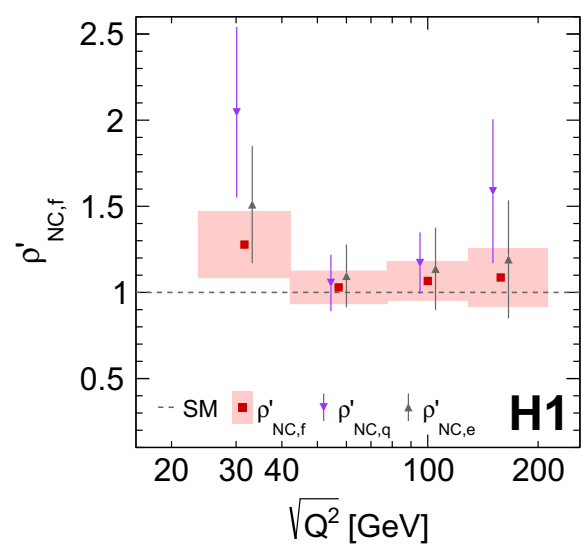

Fig. 7 Values of the $\rho_{\mathrm{NC}}^{\prime}$ and $\kappa_{\mathrm{NC}}^{\prime}$ parameters determined for four different values of $Q^{2}$. The error bars, as well as the height of the shaded areas, indicate the total uncertainties of the measurement. The width of

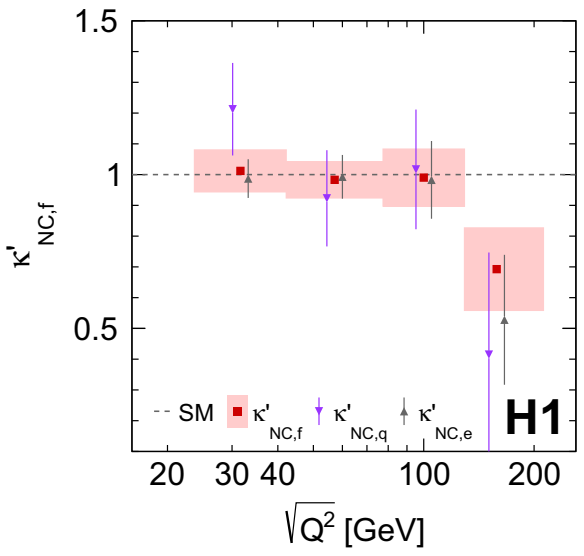

the shaded areas indicates the $Q^{2}$ range probed by the selected data. The values for the $\rho_{\mathrm{NC}, q}^{\prime}, \rho_{\mathrm{NC}, e}^{\prime}, \kappa_{\mathrm{NC}, q}^{\prime}$ and $\kappa_{\mathrm{NC}, e}^{\prime}$ parameters are horizontally displaced for better visibility 


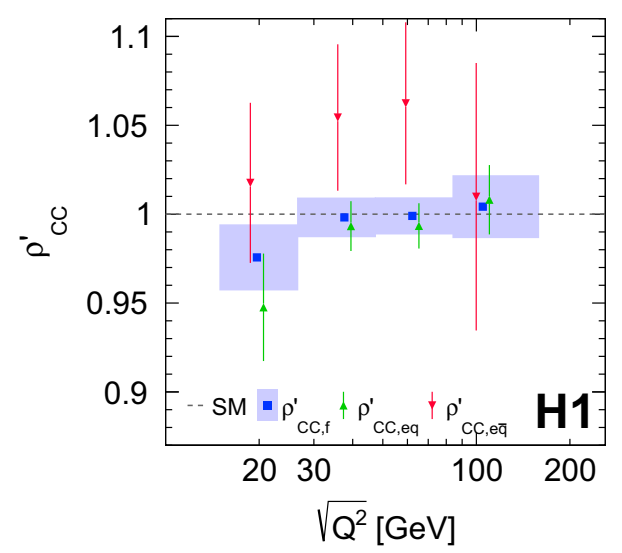

Fig. 8 Values of the $\rho_{\text {CC }}^{\prime}$ parameters determined for four different values of $Q^{2}$. The error bars, as well as the height of the shaded areas, indicate the total uncertainties of the measurement. The width of the shaded areas indicates the $Q^{2}$ range probed by the selected data. The values for the $\rho_{\mathrm{CC}, e q}^{\prime}$ and $\rho_{\mathrm{CC}, e \bar{q}}^{\prime}$ parameters are horizontally displaced for better visibility

beams leads to a substantial improvement in precision with respect to the previously published results based on the H1 HERA-I data only.

In combined electroweak and PDF fits, boson and fermion mass parameters entering cross section predictions in the onshell scheme are determined simultaneously with the parton distribution functions. The mass of the $W$ boson is determined from $\mathrm{H} 1$ data to $m_{W}=80.520 \pm 0.115 \mathrm{GeV}$, fixing $m_{Z}$ to the world average. Alternatively the $Z$-boson mass or the top-quark mass are determined with uncertainties of $110 \mathrm{MeV}$ and $26 \mathrm{GeV}$, respectively, taking $m_{W}$ to the world average. Despite their moderate precision, these results are complementary to direct measurements where particles are produced on-shell in the final state, since here the mass parameters are determined from purely virtual particle exchange only.

The axial-vector and vector weak neutral-current couplings of $u$ - and $d$-type quarks to the $Z$ boson are determined and consistency with the Standard Model expectation is observed. The axial-vector and vector couplings of the $u$ type quark are determined with a precision of about $6 \%$ and $14 \%$, respectively.

Potential modifications of the weak coupling parameters due to physics beyond the SM are studied in terms of modifications of the form factors $\rho_{\mathrm{NC}}, \kappa_{\mathrm{NC}}$ and $\rho_{\mathrm{CC}}$. For this purpose, multiplicative factors to those parameters are introduced, denoted as $\rho_{\mathrm{NC}}^{\prime}, \kappa_{\mathrm{NC}}^{\prime}$ and $\rho_{\mathrm{CC}}^{\prime}$, respectively. A precision as good as $7 \%$ or $5 \%$ of the $\rho_{\mathrm{NC}, f}^{\prime}$ and $\kappa_{\mathrm{NC}, f}^{\prime}$ parameters is achieved, respectively. The $\rho_{\mathrm{CC}}^{\prime}$ parameters are deter- mined with a precision of up to 8 per mille, and consistency with the Standard Model expectation is found. The $Q^{2}$ dependence of the $\mathrm{H} 1$ data allows for a study of the scale dependence of the $\rho_{\mathrm{NC}}^{\prime}, \kappa_{\mathrm{NC}}^{\prime}$ and $\rho_{\mathrm{CC}}^{\prime}$ parameters in the range $12<\sqrt{Q^{2}}<100 \mathrm{GeV}$, and no significant deviation from the SM expectation is observed.

Acknowledgements We are grateful to the HERA machine group whose outstanding efforts have made this experiment possible. We thank the engineers and technicians for their work in constructing and maintaining the $\mathrm{H} 1$ detector, our funding agencies for financial support, the DESY technical staff for continual assistance and the DESY directorate for support and for the hospitality which they extend to the non-DESY members of the collaboration. We would like to give credit to all partners contributing to the EGI computing infrastructure for their support for the $\mathrm{H} 1$ Collaboration. We express our thanks to all those involved in securing not only the $\mathrm{H} 1$ data but also the software and working environment for long term use, allowing the unique $\mathrm{H} 1$ data set to continue to be explored in the coming years. The transfer from experiment specific to central resources with long term support, including both storage and batch systems, has also been crucial to this enterprise. We therefore also acknowledge the role played by DESY-IT and all people involved during this transition and their future role in the years to come.

Open Access This article is distributed under the terms of the Creative Commons Attribution 4.0 International License (http://creativecomm ons.org/licenses/by/4.0/), which permits unrestricted use, distribution, and reproduction in any medium, provided you give appropriate credit to the original author(s) and the source, provide a link to the Creative Commons license, and indicate if changes were made.

Funded by SCOAP ${ }^{3}$.

\section{Appendix A: Cross section tables}

The reduced cross section measurements for NC DIS, as used in this analysis together with their systematic uncertainties [58], for different lepton beam longitudinal polarisations and for electron and positron scattering from the HERA-II running period are given in Tables 6, 7, 8 and 9, and the differential cross section for CC DIS are given in Tables 10 and 11 . The reduced cross section is related to the differential cross section, Eq. 1, by

$\sigma_{\mathrm{red}}=\frac{d^{2} \sigma^{\mathrm{NC}}}{d x d Q^{2}} \frac{x Q^{4}}{2 \pi \alpha^{2}} \frac{1}{Y_{+}}$.

The changes compared to the previously published cross sections [58] comprise the luminosity erratum [59] and the changes discussed in Sect. 3. 
Table 6 The NC $e^{-} p$ reduced cross section $\sigma_{\text {red }}$ with lepton beam polarisation $P_{e}=-25.8 \%$ with their statistical $\left(\delta_{\text {stat }}\right)$ uncertainties. The full uncertainties are available in Ref. [58], while the respective cross section values are updated according to Sect. 3 and Ref. [59]

\begin{tabular}{|c|c|c|c|c|c|c|c|c|c|c|c|}
\hline$Q^{2}\left[\mathrm{GeV}^{2}\right]$ & $x$ & $\sigma_{\text {red }}$ & $\delta_{\text {stat }}[\%]$ & $Q^{2}\left[\mathrm{GeV}^{2}\right]$ & $x$ & $\sigma_{\text {red }}$ & $\delta_{\text {stat }}[\%]$ & $Q^{2}\left[\mathrm{GeV}^{2}\right]$ & $x$ & $\sigma_{\text {red }}$ & $\delta_{\text {stat }}[\%]$ \\
\hline 120 & 0.0020 & 1.337 & 0.87 & 500 & 0.0080 & 1.010 & 2.57 & 1500 & 0.0200 & 0.8335 & 4.26 \\
\hline 120 & 0.0032 & 1.205 & 1.24 & 500 & 0.0130 & 0.9106 & 1.85 & 1500 & 0.0320 & 0.6943 & 4.06 \\
\hline 150 & 0.0032 & 1.218 & 0.73 & 500 & 0.0200 & 0.7435 & 1.83 & 1500 & 0.0500 & 0.5646 & 4.02 \\
\hline 150 & 0.0050 & 1.091 & 0.88 & 500 & 0.0320 & 0.6373 & 1.87 & 1500 & 0.0800 & 0.5143 & 4.05 \\
\hline 150 & 0.0080 & 0.9375 & 1.20 & 500 & 0.0500 & 0.5533 & 1.99 & 1500 & 0.1300 & 0.3622 & 5.25 \\
\hline 150 & 0.0130 & 0.8139 & 1.68 & 500 & 0.0800 & 0.4263 & 2.27 & 1500 & 0.1800 & 0.3159 & 5.42 \\
\hline 200 & 0.0032 & 1.247 & 1.35 & 500 & 0.1300 & 0.3740 & 2.54 & 1500 & 0.2500 & 0.2365 & 6.05 \\
\hline 200 & 0.0050 & 1.100 & 0.96 & 500 & 0.1800 & 0.3373 & 2.86 & 1500 & 0.4000 & 0.1393 & 8.82 \\
\hline 200 & 0.0080 & 0.9576 & 0.99 & 500 & 0.2500 & 0.2585 & 3.32 & 1500 & 0.6500 & 0.01511 & 14.78 \\
\hline 200 & 0.0130 & 0.7821 & 1.14 & 650 & 0.0130 & 0.9046 & 2.08 & 2000 & 0.0219 & 0.9308 & 6.58 \\
\hline 200 & 0.0200 & 0.6935 & 1.23 & 650 & 0.0200 & 0.7765 & 2.14 & 2000 & 0.0320 & 0.6562 & 4.89 \\
\hline 200 & 0.0320 & 0.5849 & 1.38 & 650 & 0.0320 & 0.6486 & 2.23 & 2000 & 0.0500 & 0.5678 & 4.87 \\
\hline 200 & 0.0500 & 0.5208 & 1.63 & 650 & 0.0500 & 0.5354 & 2.35 & 2000 & 0.0800 & 0.4520 & 5.02 \\
\hline 200 & 0.0800 & 0.4427 & 1.73 & 650 & 0.0800 & 0.4403 & 2.66 & 2000 & 0.1300 & 0.3780 & 5.98 \\
\hline 200 & 0.1300 & 0.3591 & 2.09 & 650 & 0.1300 & 0.3684 & 2.94 & 2000 & 0.1800 & 0.3071 & 6.52 \\
\hline 200 & 0.1800 & 0.3046 & 2.71 & 650 & 0.1800 & 0.3215 & 3.18 & 2000 & 0.2500 & 0.2566 & 6.68 \\
\hline 250 & 0.0050 & 1.118 & 1.12 & 650 & 0.2500 & 0.2529 & 4.13 & 2000 & 0.4000 & 0.1289 & 8.56 \\
\hline 250 & 0.0080 & 0.9705 & 1.10 & 650 & 0.4000 & 0.1251 & 6.14 & 2000 & 0.6500 & 0.01095 & 19.67 \\
\hline 250 & 0.0130 & 0.8206 & 1.20 & 800 & 0.0130 & 0.9258 & 3.50 & 3000 & 0.0320 & 0.8036 & 4.41 \\
\hline 250 & 0.0200 & 0.6944 & 1.23 & 800 & 0.0200 & 0.7391 & 2.51 & 3000 & 0.0500 & 0.6145 & 4.01 \\
\hline 250 & 0.0320 & 0.5931 & 1.30 & 800 & 0.0320 & 0.6353 & 2.67 & 3000 & 0.0800 & 0.5119 & 4.37 \\
\hline 250 & 0.0500 & 0.5069 & 1.48 & 800 & 0.0500 & 0.5523 & 2.74 & 3000 & 0.1300 & 0.4313 & 5.17 \\
\hline 250 & 0.0800 & 0.4251 & 1.52 & 800 & 0.0800 & 0.4430 & 3.04 & 3000 & 0.1800 & 0.3004 & 6.14 \\
\hline 250 & 0.1300 & 0.3632 & 1.54 & 800 & 0.1300 & 0.3476 & 3.58 & 3000 & 0.2500 & 0.2216 & 6.55 \\
\hline 250 & 0.1800 & 0.3097 & 2.11 & 800 & 0.1800 & 0.3205 & 3.75 & 3000 & 0.4000 & 0.1292 & 7.49 \\
\hline 300 & 0.0050 & 1.133 & 1.89 & 800 & 0.2500 & 0.2468 & 4.63 & 3000 & 0.6500 & 0.01350 & 14.62 \\
\hline 300 & 0.0080 & 0.9826 & 1.28 & 800 & 0.4000 & 0.1373 & 5.93 & 5000 & 0.0547 & 0.6974 & 5.98 \\
\hline 300 & 0.0130 & 0.8196 & 1.28 & 1000 & 0.0130 & 0.8664 & 3.45 & 5000 & 0.0800 & 0.5881 & 4.65 \\
\hline 300 & 0.0200 & 0.7027 & 1.42 & 1000 & 0.0200 & 0.7899 & 2.87 & 5000 & 0.1300 & 0.5103 & 5.23 \\
\hline 300 & 0.0320 & 0.5867 & 1.50 & 1000 & 0.0320 & 0.6760 & 2.82 & 5000 & 0.1800 & 0.3976 & 6.13 \\
\hline 300 & 0.0500 & 0.4994 & 1.62 & 1000 & 0.0500 & 0.5166 & 3.15 & 5000 & 0.2500 & 0.2348 & 8.02 \\
\hline 300 & 0.0800 & 0.4250 & 1.72 & 1000 & 0.0800 & 0.4428 & 3.43 & 5000 & 0.4000 & 0.1101 & 9.88 \\
\hline 300 & 0.1300 & 0.3621 & 1.71 & 1000 & 0.1300 & 0.3396 & 4.21 & 5000 & 0.6500 & 0.01502 & 16.48 \\
\hline 300 & 0.1800 & 0.3023 & 2.26 & 1000 & 0.1800 & 0.3682 & 3.98 & 8000 & 0.0875 & 0.6943 & 8.89 \\
\hline 300 & 0.4000 & 0.1468 & 2.75 & 1000 & 0.2500 & 0.2659 & 4.61 & 8000 & 0.1300 & 0.5661 & 7.10 \\
\hline 400 & 0.0080 & 1.048 & 1.54 & 1000 & 0.4000 & 0.1299 & 6.56 & 8000 & 0.1800 & 0.4017 & 8.01 \\
\hline 400 & 0.0130 & 0.8622 & 1.50 & 1200 & 0.0130 & 0.9440 & 5.43 & 8000 & 0.2500 & 0.2807 & 9.07 \\
\hline 400 & 0.0200 & 0.7260 & 1.54 & 1200 & 0.0200 & 0.7891 & 3.60 & 8000 & 0.4000 & 0.1232 & 12.62 \\
\hline 400 & 0.0320 & 0.6114 & 1.63 & 1200 & 0.0320 & 0.6964 & 3.27 & 8000 & 0.6500 & 0.01091 & 21.89 \\
\hline 400 & 0.0500 & 0.4951 & 1.84 & 1200 & 0.0500 & 0.5465 & 3.48 & 12,000 & 0.1300 & 0.7921 & 15.45 \\
\hline 400 & 0.0800 & 0.4279 & 1.91 & 1200 & 0.0800 & 0.4591 & 3.73 & 12,000 & 0.1800 & 0.5805 & 9.59 \\
\hline 400 & 0.1300 & 0.3676 & 1.93 & 1200 & 0.1300 & 0.3602 & 5.38 & 12,000 & 0.2500 & 0.3347 & 11.15 \\
\hline 400 & 0.1800 & 0.3055 & 2.43 & 1200 & 0.1800 & 0.3308 & 4.65 & 12,000 & 0.4000 & 0.2244 & 12.42 \\
\hline \multirow[t]{2}{*}{400} & 0.4000 & 0.1469 & 3.09 & 1200 & 0.2500 & 0.2207 & 5.58 & 12,000 & 0.6500 & 0.01526 & 27.80 \\
\hline & & & & 1200 & 0.4000 & 0.1264 & 7.08 & 20,000 & 0.2500 & 0.6549 & 13.34 \\
\hline
\end{tabular}


Table 6 continued

\begin{tabular}{|c|c|c|c|c|c|c|c|c|c|c|c|}
\hline$Q^{2}\left[\mathrm{GeV}^{2}\right]$ & $x$ & $\sigma_{\text {red }}$ & $\delta_{\text {stat }}[\%]$ & $Q^{2}\left[\mathrm{GeV}^{2}\right]$ & $x$ & $\sigma_{\text {red }}$ & $\delta_{\text {stat }}[\%]$ & $Q^{2}\left[\mathrm{GeV}^{2}\right]$ & $x$ & $\sigma_{\text {red }}$ & $\delta_{\text {stat }}[\%]$ \\
\hline & & & & & & & & 20,000 & 0.4000 & 0.2329 & 16.55 \\
\hline & & & & & & & & 20,000 & 0.6500 & 0.01985 & 40.89 \\
\hline & & & & & & & & 30,000 & 0.4000 & 0.1845 & 36.01 \\
\hline & & & & & & & & 30,000 & 0.6500 & 0.04510 & 37.83 \\
\hline & & & & & & & & 50,000 & 0.6500 & 0.1250 & 57.78 \\
\hline
\end{tabular}

Table 7 The NC $e^{-} p$ reduced cross section $\sigma_{\text {red }}$ with lepton beam polarisation $P_{e}=36.0 \%$ with their statistical $\left(\delta_{\text {stat }}\right)$ uncertainties. The full uncertainties are available in Ref. [58], while the respective cross section values are updated according to Sect. 3 and Ref. [59]

\begin{tabular}{|c|c|c|c|c|c|c|c|c|c|c|c|}
\hline$Q^{2}\left[\mathrm{GeV}^{2}\right]$ & $x$ & $\sigma_{\text {red }}$ & $\delta_{\text {stat }}[\%]$ & $Q^{2}\left[\mathrm{GeV}^{2}\right]$ & $x$ & $\sigma_{\text {red }}$ & $\delta_{\text {stat }}[\%]$ & $Q^{2}\left[\mathrm{GeV}^{2}\right]$ & $x$ & $\sigma_{\text {red }}$ & $\overline{\delta_{\text {stat }}[\%]}$ \\
\hline 120 & 0.0020 & 1.340 & 1.29 & 500 & 0.0080 & 0.9586 & 3.93 & 1500 & 0.0200 & 0.7317 & 6.68 \\
\hline 120 & 0.0032 & 1.213 & 1.78 & 500 & 0.0130 & 0.8227 & 2.80 & 1500 & 0.0320 & 0.6439 & 6.22 \\
\hline 150 & 0.0032 & 1.208 & 1.09 & 500 & 0.0200 & 0.6873 & 2.80 & 1500 & 0.0500 & 0.5514 & 6.00 \\
\hline 150 & 0.0050 & 1.104 & 1.29 & 500 & 0.0320 & 0.5849 & 2.89 & 1500 & 0.0800 & 0.4600 & 6.35 \\
\hline 150 & 0.0080 & 0.9534 & 1.78 & 500 & 0.0500 & 0.5161 & 3.01 & 1500 & 0.1300 & 0.3344 & 10.17 \\
\hline 150 & 0.0130 & 0.7840 & 2.42 & 500 & 0.0800 & 0.4334 & 3.28 & 1500 & 0.1800 & 0.2695 & 8.79 \\
\hline 200 & 0.0032 & 1.189 & 2.06 & 500 & 0.1300 & 0.3687 & 4.14 & 1500 & 0.2500 & 0.2555 & 8.68 \\
\hline 200 & 0.0050 & 1.092 & 1.46 & 500 & 0.1800 & 0.3218 & 4.06 & 1500 & 0.4000 & 0.09316 & 13.16 \\
\hline 200 & 0.0080 & 0.9487 & 1.44 & 500 & 0.2500 & 0.2447 & 5.05 & 1500 & 0.6500 & 0.01262 & 23.63 \\
\hline 200 & 0.0130 & 0.7938 & 1.67 & 650 & 0.0130 & 0.8753 & 3.13 & 2000 & 0.0219 & 0.7628 & 10.62 \\
\hline 200 & 0.0200 & 0.6910 & 1.81 & 650 & 0.0200 & 0.7334 & 3.26 & 2000 & 0.0320 & 0.6464 & 7.29 \\
\hline 200 & 0.0320 & 0.5630 & 2.12 & 650 & 0.0320 & 0.6383 & 3.33 & 2000 & 0.0500 & 0.5190 & 7.57 \\
\hline 200 & 0.0500 & 0.5323 & 2.48 & 650 & 0.0500 & 0.5511 & 3.46 & 2000 & 0.0800 & 0.4552 & 7.37 \\
\hline 200 & 0.0800 & 0.4308 & 2.51 & 650 & 0.0800 & 0.4102 & 4.01 & 2000 & 0.1300 & 0.3166 & 9.69 \\
\hline 200 & 0.1300 & 0.3616 & 2.84 & 650 & 0.1300 & 0.3354 & 4.99 & 2000 & 0.1800 & 0.2939 & 9.83 \\
\hline 200 & 0.1800 & 0.3113 & 4.12 & 650 & 0.1800 & 0.3324 & 4.67 & 2000 & 0.2500 & 0.2322 & 10.39 \\
\hline 250 & 0.0050 & 1.100 & 1.69 & 650 & 0.2500 & 0.2521 & 5.55 & 2000 & 0.4000 & 0.1216 & 12.93 \\
\hline 250 & 0.0080 & 0.9277 & 1.64 & 650 & 0.4000 & 0.1130 & 8.49 & 2000 & 0.6500 & 0.008022 & 33.44 \\
\hline 250 & 0.0130 & 0.7978 & 1.80 & 800 & 0.0130 & 0.8344 & 5.20 & 3000 & 0.0320 & 0.6126 & 7.46 \\
\hline 250 & 0.0200 & 0.6690 & 1.86 & 800 & 0.0200 & 0.7130 & 3.76 & 3000 & 0.0500 & 0.6022 & 5.96 \\
\hline 250 & 0.0320 & 0.5657 & 1.95 & 800 & 0.0320 & 0.6115 & 3.87 & 3000 & 0.0800 & 0.4925 & 6.63 \\
\hline 250 & 0.0500 & 0.4677 & 2.20 & 800 & 0.0500 & 0.5470 & 4.04 & 3000 & 0.1300 & 0.3542 & 8.44 \\
\hline 250 & 0.0800 & 0.4305 & 2.24 & 800 & 0.0800 & 0.3842 & 4.83 & 3000 & 0.1800 & 0.3105 & 9.00 \\
\hline 250 & 0.1300 & 0.3710 & 2.29 & 800 & 0.1300 & 0.3592 & 5.90 & 3000 & 0.2500 & 0.2919 & 8.59 \\
\hline 250 & 0.1800 & 0.3035 & 3.24 & 800 & 0.1800 & 0.3187 & 6.28 & 3000 & 0.4000 & 0.09196 & 12.93 \\
\hline 300 & 0.0050 & 1.163 & 2.77 & 800 & 0.2500 & 0.2272 & 6.66 & 3000 & 0.6500 & 0.005166 & 35.57 \\
\hline 300 & 0.0080 & 0.9754 & 1.89 & 800 & 0.4000 & 0.1210 & 9.46 & 5000 & 0.0547 & 0.5881 & 9.54 \\
\hline 300 & 0.0130 & 0.8091 & 1.92 & 1000 & 0.0130 & 0.8399 & 5.19 & 5000 & 0.0800 & 0.4575 & 7.68 \\
\hline 300 & 0.0200 & 0.6930 & 2.10 & 1000 & 0.0200 & 0.7135 & 4.48 & 5000 & 0.1300 & 0.4144 & 8.49 \\
\hline 300 & 0.0320 & 0.5937 & 2.18 & 1000 & 0.0320 & 0.6349 & 4.66 & 5000 & 0.1800 & 0.3602 & 9.47 \\
\hline 300 & 0.0500 & 0.5014 & 2.46 & 1000 & 0.0500 & 0.5027 & 4.74 & 5000 & 0.2500 & 0.2529 & 16.81 \\
\hline 300 & 0.0800 & 0.4269 & 2.56 & 1000 & 0.0800 & 0.4182 & 5.21 & 5000 & 0.4000 & 0.1434 & 12.82 \\
\hline 300 & 0.1300 & 0.3530 & 2.61 & 1000 & 0.1300 & 0.3902 & 5.82 & 5000 & 0.6500 & 0.01324 & 25.88 \\
\hline 300 & 0.1800 & 0.2847 & 3.47 & 1000 & 0.1800 & 0.3002 & 6.43 & 8000 & 0.0875 & 0.6279 & 13.94 \\
\hline 300 & 0.4000 & 0.1523 & 3.91 & 1000 & 0.2500 & 0.2774 & 6.71 & 8000 & 0.1300 & 0.4992 & 11.13 \\
\hline 400 & 0.0080 & 0.9979 & 2.41 & 1000 & 0.4000 & 0.1267 & 9.92 & 8000 & 0.1800 & 0.3997 & 11.74 \\
\hline
\end{tabular}


Table 7 continued

\begin{tabular}{|c|c|c|c|c|c|c|c|c|c|c|c|}
\hline$Q^{2}\left[\mathrm{GeV}^{2}\right]$ & $x$ & $\sigma_{\text {red }}$ & $\delta_{\text {stat }}[\%]$ & $Q^{2}\left[\mathrm{GeV}^{2}\right]$ & $x$ & $\sigma_{\text {red }}$ & $\delta_{\text {stat }}[\%]$ & $Q^{2}\left[\mathrm{GeV}^{2}\right]$ & $x$ & $\sigma_{\text {red }}$ & $\overline{\delta_{\text {stat }}[\%]}$ \\
\hline 400 & 0.0130 & 0.8314 & 2.24 & 1200 & 0.0130 & 0.7777 & 9.00 & 8000 & 0.2500 & 0.2553 & 14.04 \\
\hline 400 & 0.0200 & 0.6742 & 2.36 & 1200 & 0.0200 & 0.7689 & 5.37 & 8000 & 0.4000 & 0.1182 & 18.93 \\
\hline 400 & 0.0320 & 0.5909 & 2.46 & 1200 & 0.0320 & 0.6439 & 5.03 & 8000 & 0.6500 & 0.01682 & 26.77 \\
\hline 400 & 0.0500 & 0.4953 & 2.70 & 1200 & 0.0500 & 0.5285 & 5.22 & 12,000 & 0.1300 & 0.7385 & 23.42 \\
\hline 400 & 0.0800 & 0.3995 & 3.05 & 1200 & 0.0800 & 0.4649 & 5.53 & 12,000 & 0.1800 & 0.4153 & 16.73 \\
\hline 400 & 0.1300 & 0.3666 & 2.96 & 1200 & 0.1300 & 0.3395 & 7.00 & 12,000 & 0.2500 & 0.3198 & 16.72 \\
\hline 400 & 0.1800 & 0.3074 & 3.45 & 1200 & 0.1800 & 0.2714 & 7.60 & 12,000 & 0.4000 & 0.1575 & 21.86 \\
\hline \multirow[t]{6}{*}{400} & 0.4000 & 0.1482 & 4.99 & 1200 & 0.2500 & 0.2206 & 8.26 & 12,000 & 0.6500 & 0.01281 & 44.83 \\
\hline & & & & 1200 & 0.4000 & 0.1337 & 10.01 & 20,000 & 0.2500 & 0.2146 & 34.11 \\
\hline & & & & & & & & 20,000 & 0.4000 & 0.2378 & 24.30 \\
\hline & & & & & & & & 20,000 & 0.6500 & 0.01372 & 70.89 \\
\hline & & & & & & & & 30,000 & 0.4000 & 0.2765 & 43.40 \\
\hline & & & & & & & & 30,000 & 0.6500 & 0.04110 & 57.81 \\
\hline
\end{tabular}

Table 8 The $\mathrm{NC} e^{+} p$ reduced cross section $\sigma_{\text {red }}$ with lepton beam polarisation $P_{e}=-37.0 \%$ with their statistical $\left(\delta_{\text {stat }}\right)$ uncertainties. The full uncertainties are available in Ref. [58], while the respective cross section values are updated according to Sect. 3 and Ref. [59]

\begin{tabular}{|c|c|c|c|c|c|c|c|c|c|c|c|}
\hline$Q^{2}\left[\mathrm{GeV}^{2}\right]$ & $x$ & $\sigma_{\text {red }}$ & $\delta_{\text {stat }}[\%]$ & $Q^{2}\left[\mathrm{GeV}^{2}\right]$ & $x$ & $\sigma_{\text {red }}$ & $\delta_{\text {stat }}[\%]$ & $Q^{2}\left[\mathrm{GeV}^{2}\right]$ & $x$ & $\sigma_{\text {red }}$ & $\overline{\delta_{\text {stat }}[\%]}$ \\
\hline 120 & 0.0020 & 1.367 & 0.97 & 500 & 0.0080 & 0.9862 & 2.93 & 1500 & 0.0200 & 0.6695 & 5.31 \\
\hline 120 & 0.0032 & 1.249 & 1.38 & 500 & 0.0130 & 0.8805 & 2.10 & 1500 & 0.0320 & 0.5980 & 5.22 \\
\hline 150 & 0.0032 & 1.248 & 0.82 & 500 & 0.0200 & 0.7446 & 2.09 & 1500 & 0.0500 & 0.5295 & 4.55 \\
\hline 150 & 0.0050 & 1.096 & 1.00 & 500 & 0.0320 & 0.6097 & 2.28 & 1500 & 0.0800 & 0.4702 & 5.12 \\
\hline 150 & 0.0080 & 0.9470 & 1.36 & 500 & 0.0500 & 0.5252 & 2.29 & 1500 & 0.1300 & 0.3057 & 6.35 \\
\hline 150 & 0.0130 & 0.8224 & 1.92 & 500 & 0.0800 & 0.4306 & 2.48 & 1500 & 0.1800 & 0.2927 & 6.32 \\
\hline 200 & 0.0032 & 1.263 & 1.55 & 500 & 0.1300 & 0.4018 & 2.93 & 1500 & 0.2500 & 0.2585 & 6.39 \\
\hline 200 & 0.0050 & 1.122 & 1.08 & 500 & 0.1800 & 0.3160 & 3.13 & 1500 & 0.4000 & 0.1211 & 8.89 \\
\hline 200 & 0.0080 & 0.9667 & 1.09 & 500 & 0.2500 & 0.2502 & 3.83 & 1500 & 0.6500 & 0.01573 & 16.04 \\
\hline 200 & 0.0130 & 0.8071 & 1.24 & 650 & 0.0130 & 0.8789 & 2.35 & 2000 & 0.0219 & 0.6690 & 8.55 \\
\hline 200 & 0.0200 & 0.7003 & 1.38 & 650 & 0.0200 & 0.7456 & 2.47 & 2000 & 0.0320 & 0.5502 & 5.99 \\
\hline 200 & 0.0320 & 0.5918 & 1.61 & 650 & 0.0320 & 0.6240 & 2.56 & 2000 & 0.0500 & 0.5168 & 5.63 \\
\hline 200 & 0.0500 & 0.5312 & 1.79 & 650 & 0.0500 & 0.5102 & 2.74 & 2000 & 0.0800 & 0.4365 & 5.55 \\
\hline 200 & 0.0800 & 0.4385 & 1.99 & 650 & 0.0800 & 0.4037 & 3.04 & 2000 & 0.1300 & 0.3138 & 7.22 \\
\hline 200 & 0.1300 & 0.3722 & 2.25 & 650 & 0.1300 & 0.3624 & 3.30 & 2000 & 0.1800 & 0.2954 & 7.37 \\
\hline 200 & 0.1800 & 0.3266 & 2.97 & 650 & 0.1800 & 0.3269 & 3.57 & 2000 & 0.2500 & 0.2150 & 7.85 \\
\hline 250 & 0.0050 & 1.128 & 1.25 & 650 & 0.2500 & 0.2449 & 4.65 & 2000 & 0.4000 & 0.1188 & 9.92 \\
\hline 250 & 0.0080 & 0.9659 & 1.24 & 650 & 0.4000 & 0.1366 & 6.70 & 2000 & 0.6500 & 0.01324 & 19.28 \\
\hline 250 & 0.0130 & 0.8085 & 1.38 & 800 & 0.0130 & 0.7990 & 3.94 & 3000 & 0.0320 & 0.5883 & 5.61 \\
\hline 250 & 0.0200 & 0.6896 & 1.40 & 800 & 0.0200 & 0.7034 & 2.84 & 3000 & 0.0500 & 0.4774 & 5.02 \\
\hline 250 & 0.0320 & 0.5789 & 1.46 & 800 & 0.0320 & 0.5953 & 3.09 & 3000 & 0.0800 & 0.4114 & 5.36 \\
\hline 250 & 0.0500 & 0.5079 & 1.57 & 800 & 0.0500 & 0.5276 & 3.14 & 3000 & 0.1300 & 0.3340 & 6.38 \\
\hline 250 & 0.0800 & 0.4438 & 1.71 & 800 & 0.0800 & 0.4697 & 3.35 & 3000 & 0.1800 & 0.2711 & 7.11 \\
\hline 250 & 0.1300 & 0.3836 & 1.81 & 800 & 0.1300 & 0.3511 & 4.04 & 3000 & 0.2500 & 0.2219 & 7.07 \\
\hline 250 & 0.1800 & 0.3011 & 2.39 & 800 & 0.1800 & 0.3237 & 4.18 & 3000 & 0.4000 & 0.1272 & 8.29 \\
\hline
\end{tabular}


Table 8 continued

\begin{tabular}{|c|c|c|c|c|c|c|c|c|c|c|c|}
\hline$Q^{2}\left[\mathrm{GeV}^{2}\right]$ & $x$ & $\sigma_{\text {red }}$ & $\delta_{\text {stat }}[\%]$ & $Q^{2}\left[\mathrm{GeV}^{2}\right]$ & $x$ & $\sigma_{\text {red }}$ & $\delta_{\text {stat }}[\%]$ & $Q^{2}\left[\mathrm{GeV}^{2}\right]$ & $x$ & $\sigma_{\text {red }}$ & $\delta_{\text {stat }}[\%]$ \\
\hline 300 & 0.0050 & 1.135 & 2.13 & 800 & 0.2500 & 0.2226 & 5.17 & 3000 & 0.6500 & 0.01302 & 16.94 \\
\hline 300 & 0.0080 & 0.9749 & 1.45 & 800 & 0.4000 & 0.1247 & 7.21 & 5000 & 0.0547 & 0.4324 & 7.86 \\
\hline 300 & 0.0130 & 0.8181 & 1.45 & 1000 & 0.0130 & 0.8326 & 3.89 & 5000 & 0.0800 & 0.3520 & 6.38 \\
\hline 300 & 0.0200 & 0.7086 & 1.60 & 1000 & 0.0200 & 0.7443 & 3.28 & 5000 & 0.1300 & 0.3150 & 7.32 \\
\hline 300 & 0.0320 & 0.5926 & 1.69 & 1000 & 0.0320 & 0.5882 & 3.38 & 5000 & 0.1800 & 0.2647 & 8.19 \\
\hline 300 & 0.0500 & 0.5053 & 1.82 & 1000 & 0.0500 & 0.5003 & 3.58 & 5000 & 0.2500 & 0.2278 & 8.92 \\
\hline 300 & 0.0800 & 0.4462 & 1.85 & 1000 & 0.0800 & 0.4275 & 3.88 & 5000 & 0.4000 & 0.09719 & 11.65 \\
\hline 300 & 0.1300 & 0.3717 & 1.93 & 1000 & 0.1300 & 0.3378 & 4.75 & 5000 & 0.6500 & 0.007011 & 27.87 \\
\hline 300 & 0.1800 & 0.3081 & 2.52 & 1000 & 0.1800 & 0.3008 & 4.92 & 8000 & 0.0875 & 0.2552 & 15.58 \\
\hline 300 & 0.4000 & 0.1551 & 3.06 & 1000 & 0.2500 & 0.2354 & 5.56 & 8000 & 0.1300 & 0.2586 & 11.14 \\
\hline 400 & 0.0080 & 1.025 & 1.77 & 1000 & 0.4000 & 0.1210 & 7.75 & 8000 & 0.1800 & 0.2346 & 11.31 \\
\hline 400 & 0.0130 & 0.8345 & 1.71 & 1200 & 0.0130 & 0.7975 & 6.75 & 8000 & 0.2500 & 0.2234 & 11.01 \\
\hline 400 & 0.0200 & 0.7131 & 1.77 & 1200 & 0.0200 & 0.6749 & 4.32 & 8000 & 0.4000 & 0.1034 & 15.10 \\
\hline 400 & 0.0320 & 0.6080 & 1.91 & 1200 & 0.0320 & 0.6406 & 3.76 & 8000 & 0.6500 & 0.01192 & 25.07 \\
\hline 400 & 0.0500 & 0.5019 & 2.05 & 1200 & 0.0500 & 0.5253 & 3.95 & 12,000 & 0.1300 & 0.2033 & 28.52 \\
\hline 400 & 0.0800 & 0.4265 & 2.15 & 1200 & 0.0800 & 0.4256 & 4.33 & 12,000 & 0.1800 & 0.2078 & 17.53 \\
\hline 400 & 0.1300 & 0.3662 & 2.11 & 1200 & 0.1300 & 0.3242 & 5.38 & 12,000 & 0.2500 & 0.1426 & 18.70 \\
\hline 400 & 0.1800 & 0.3066 & 2.76 & 1200 & 0.1800 & 0.2971 & 5.47 & 12,000 & 0.4000 & 0.07284 & 24.35 \\
\hline \multirow[t]{4}{*}{400} & 0.4000 & 0.1572 & 3.56 & 1200 & 0.2500 & 0.2680 & 5.62 & 12,000 & 0.6500 & 0.008088 & 44.93 \\
\hline & & & & 1200 & 0.4000 & 0.1086 & 8.59 & 20,000 & 0.2500 & 0.1039 & 32.86 \\
\hline & & & & & & & & 20,000 & 0.4000 & 0.07670 & 31.82 \\
\hline & & & & & & & & 20,000 & 0.6500 & 0.01353 & 57.87 \\
\hline
\end{tabular}

Table 9 The $\mathrm{NC} e^{+} p$ reduced cross section $\sigma_{\text {red }}$ with lepton beam polarisation $P_{e}=32.5 \%$ with their statistical $\left(\delta_{\text {stat }}\right)$ uncertainties. The full uncertainties are available in Ref. [58], while the respective cross section values are updated according to Sect. 3 and Ref. [59]

\begin{tabular}{|c|c|c|c|c|c|c|c|c|c|c|c|}
\hline$Q^{2}\left[\mathrm{GeV}^{2}\right]$ & $x$ & $\sigma_{\text {red }}$ & $\delta_{\text {stat }}[\%]$ & $Q^{2}\left[\mathrm{GeV}^{2}\right]$ & $x$ & $\sigma_{\text {red }}$ & $\delta_{\text {stat }}[\%]$ & $Q^{2}\left[\mathrm{GeV}^{2}\right]$ & $x$ & $\sigma_{\text {red }}$ & $\delta_{\text {stat }}[\%]$ \\
\hline 120 & 0.0020 & 1.353 & 0.87 & 500 & 0.0080 & 0.9862 & 2.69 & 1500 & 0.0200 & 0.7066 & 4.63 \\
\hline 120 & 0.0032 & 1.192 & 1.27 & 500 & 0.0130 & 0.8622 & 1.85 & 1500 & 0.0320 & 0.6057 & 4.29 \\
\hline 150 & 0.0032 & 1.224 & 0.74 & 500 & 0.0200 & 0.7448 & 1.89 & 1500 & 0.0500 & 0.5409 & 4.00 \\
\hline 150 & 0.0050 & 1.096 & 0.88 & 500 & 0.0320 & 0.6130 & 1.95 & 1500 & 0.0800 & 0.4435 & 4.31 \\
\hline 150 & 0.0080 & 0.9530 & 1.22 & 500 & 0.0500 & 0.5351 & 2.06 & 1500 & 0.1300 & 0.3634 & 5.16 \\
\hline 150 & 0.0130 & 0.7836 & 1.71 & 500 & 0.0800 & 0.4512 & 2.21 & 1500 & 0.1800 & 0.3161 & 5.38 \\
\hline 200 & 0.0032 & 1.225 & 1.40 & 500 & 0.1300 & 0.3739 & 2.49 & 1500 & 0.2500 & 0.2148 & 6.22 \\
\hline 200 & 0.0050 & 1.094 & 0.97 & 500 & 0.1800 & 0.3124 & 2.91 & 1500 & 0.4000 & 0.1278 & 7.55 \\
\hline 200 & 0.0080 & 0.9510 & 0.98 & 500 & 0.2500 & 0.2508 & 3.60 & 1500 & 0.6500 & 0.01479 & 14.78 \\
\hline 200 & 0.0130 & 0.7985 & 1.11 & 650 & 0.0130 & 0.8444 & 2.14 & 2000 & 0.0219 & 0.7342 & 7.48 \\
\hline 200 & 0.0200 & 0.6889 & 1.22 & 650 & 0.0200 & 0.7301 & 2.21 & 2000 & 0.0320 & 0.5603 & 5.24 \\
\hline 200 & 0.0320 & 0.5832 & 1.40 & 650 & 0.0320 & 0.6681 & 2.28 & 2000 & 0.0500 & 0.5596 & 4.83 \\
\hline 200 & 0.0500 & 0.5022 & 1.62 & 650 & 0.0500 & 0.5319 & 2.38 & 2000 & 0.0800 & 0.4293 & 5.03 \\
\hline 200 & 0.0800 & 0.4385 & 1.77 & 650 & 0.0800 & 0.4372 & 2.68 & 2000 & 0.1300 & 0.3821 & 6.71 \\
\hline 200 & 0.1300 & 0.3558 & 1.96 & 650 & 0.1300 & 0.3882 & 3.20 & 2000 & 0.1800 & 0.3152 & 6.34 \\
\hline 200 & 0.1800 & 0.3053 & 2.68 & 650 & 0.1800 & 0.3478 & 3.07 & 2000 & 0.2500 & 0.2608 & 6.45 \\
\hline 250 & 0.0050 & 1.124 & 1.13 & 650 & 0.2500 & 0.2389 & 3.85 & 2000 & 0.4000 & 0.1368 & 8.15 \\
\hline 250 & 0.0080 & 0.9603 & 1.10 & 650 & 0.4000 & 0.1352 & 5.35 & 2000 & 0.6500 & 0.01480 & 17.19 \\
\hline 250 & 0.0130 & 0.8134 & 1.22 & 800 & 0.0130 & 0.8458 & 3.47 & 3000 & 0.0320 & 0.6145 & 5.01 \\
\hline
\end{tabular}


Table 9 continued

\begin{tabular}{|c|c|c|c|c|c|c|c|c|c|c|c|}
\hline$Q^{2}\left[\mathrm{GeV}^{2}\right]$ & $x$ & $\sigma_{\text {red }}$ & $\delta_{\text {stat }}[\%]$ & $Q^{2}\left[\mathrm{GeV}^{2}\right]$ & $x$ & $\sigma_{\text {red }}$ & $\delta_{\text {stat }}[\%]$ & $Q^{2}\left[\mathrm{GeV}^{2}\right]$ & $x$ & $\sigma_{\text {red }}$ & $\delta_{\text {stat }}[\%]$ \\
\hline 250 & 0.0200 & 0.7022 & 1.25 & 800 & 0.0200 & 0.7083 & 2.52 & 3000 & 0.0500 & 0.5424 & 4.22 \\
\hline 250 & 0.0320 & 0.5830 & 1.31 & 800 & 0.0320 & 0.6392 & 2.60 & 3000 & 0.0800 & 0.4717 & 4.45 \\
\hline 250 & 0.0500 & 0.5018 & 1.45 & 800 & 0.0500 & 0.5330 & 2.90 & 3000 & 0.1300 & 0.3559 & 5.53 \\
\hline 250 & 0.0800 & 0.4335 & 1.46 & 800 & 0.0800 & 0.4504 & 3.06 & 3000 & 0.1800 & 0.3364 & 7.17 \\
\hline 250 & 0.1300 & 0.3587 & 1.60 & 800 & 0.1300 & 0.3663 & 3.54 & 3000 & 0.2500 & 0.2359 & 6.20 \\
\hline 250 & 0.1800 & 0.2972 & 2.20 & 800 & 0.1800 & 0.3316 & 3.68 & 3000 & 0.4000 & 0.1200 & 7.64 \\
\hline 300 & 0.0050 & 1.140 & 1.94 & 800 & 0.2500 & 0.2574 & 4.23 & 3000 & 0.6500 & 0.01293 & 15.66 \\
\hline 300 & 0.0080 & 0.9790 & 1.29 & 800 & 0.4000 & 0.1215 & 6.45 & 5000 & 0.0547 & 0.5109 & 6.66 \\
\hline 300 & 0.0130 & 0.8001 & 1.31 & 1000 & 0.0130 & 0.7831 & 3.64 & 5000 & 0.0800 & 0.4688 & 4.98 \\
\hline 300 & 0.0200 & 0.7169 & 1.44 & 1000 & 0.0200 & 0.7302 & 2.97 & 5000 & 0.1300 & 0.3724 & 5.99 \\
\hline 300 & 0.0320 & 0.5788 & 1.51 & 1000 & 0.0320 & 0.6470 & 2.86 & 5000 & 0.1800 & 0.3302 & 6.59 \\
\hline 300 & 0.0500 & 0.4936 & 1.66 & 1000 & 0.0500 & 0.5420 & 3.09 & 5000 & 0.2500 & 0.2143 & 8.44 \\
\hline 300 & 0.0800 & 0.4384 & 1.71 & 1000 & 0.0800 & 0.4554 & 3.40 & 5000 & 0.4000 & 0.1151 & 9.78 \\
\hline 300 & 0.1300 & 0.3724 & 1.75 & 1000 & 0.1300 & 0.3484 & 4.49 & 5000 & 0.6500 & 0.01243 & 18.62 \\
\hline 300 & 0.1800 & 0.3087 & 2.26 & 1000 & 0.1800 & 0.3044 & 4.35 & 8000 & 0.0875 & 0.4324 & 10.53 \\
\hline 300 & 0.4000 & 0.1476 & 2.95 & 1000 & 0.2500 & 0.2559 & 4.74 & 8000 & 0.1300 & 0.3196 & 9.01 \\
\hline 400 & 0.0080 & 0.9859 & 1.60 & 1000 & 0.4000 & 0.1382 & 8.52 & 8000 & 0.1800 & 0.2936 & 9.01 \\
\hline 400 & 0.0130 & 0.8665 & 1.49 & 1200 & 0.0130 & 0.8759 & 5.78 & 8000 & 0.2500 & 0.2262 & 13.15 \\
\hline 400 & 0.0200 & 0.7125 & 1.57 & 1200 & 0.0200 & 0.7496 & 3.64 & 8000 & 0.4000 & 0.1021 & 13.63 \\
\hline 400 & 0.0320 & 0.5910 & 1.68 & 1200 & 0.0320 & 0.5929 & 3.51 & 8000 & 0.6500 & 0.01562 & 19.28 \\
\hline 400 & 0.0500 & 0.4989 & 1.85 & 1200 & 0.0500 & 0.5162 & 3.52 & 12,000 & 0.1300 & 0.2127 & 27.73 \\
\hline 400 & 0.0800 & 0.4340 & 2.00 & 1200 & 0.0800 & 0.4456 & 3.76 & 12,000 & 0.1800 & 0.2220 & 15.03 \\
\hline 400 & 0.1300 & 0.3538 & 1.94 & 1200 & 0.1300 & 0.3656 & 4.55 & 12,000 & 0.2500 & 0.1707 & 15.33 \\
\hline 400 & 0.1800 & 0.3076 & 2.51 & 1200 & 0.1800 & 0.3449 & 5.25 & 12,000 & 0.4000 & 0.1257 & 16.94 \\
\hline \multirow[t]{6}{*}{400} & 0.4000 & 0.1435 & 3.13 & 1200 & 0.2500 & 0.2404 & 5.97 & 12,000 & 0.6500 & 0.02261 & 24.29 \\
\hline & & & & 1200 & 0.4000 & 0.1103 & 7.57 & 20,000 & 0.2500 & 0.1423 & 25.06 \\
\hline & & & & & & & & 20,000 & 0.4000 & 0.1118 & 23.67 \\
\hline & & & & & & & & 20,000 & 0.6500 & 0.006952 & 71.00 \\
\hline & & & & & & & & 30,000 & 0.4000 & 0.07828 & 51.28 \\
\hline & & & & & & & & 30,000 & 0.6500 & 0.01392 & 70.94 \\
\hline
\end{tabular}

Table 10 The $\mathrm{CC} e^{-} p$ cross section $\sigma=d^{2} \sigma^{\mathrm{CC}} / d x d Q^{2}$ for lepton polarisation

$P_{e}=-25.8 \%$ (left) and

$P_{e}=36.0 \%$ (right) with their

statistical $\left(\delta_{\text {stat }}\right)$ uncertainties.

The full uncertainties are

available in Ref. [58], while the

respective cross section values

are updated according to Sect. 3 and Ref. [59]

\begin{tabular}{lllcclll}
\hline$Q^{2}\left[\mathrm{GeV}^{2}\right]$ & $x$ & $\sigma\left[\mathrm{pb} / \mathrm{GeV}^{2}\right]$ & $\delta_{\text {stat }}[\%]$ & $Q^{2}\left[\mathrm{GeV}^{2}\right]$ & $x$ & $\sigma\left[\mathrm{pb} / \mathrm{GeV}^{2}\right]$ & $\delta_{\text {stat }}[\%]$ \\
\hline 300 & 0.008 & 2.03 & 40.6 & 300 & 0.008 & 1.18 & 47.2 \\
300 & 0.013 & 0.934 & 14.4 & 300 & 0.013 & 0.428 & 35.0 \\
300 & 0.032 & 0.309 & 14.0 & 300 & 0.032 & 0.129 & 24.9 \\
300 & 0.080 & $0.785 \times 10^{-1}$ & 13.5 & 300 & 0.080 & $0.473 \times 10^{-1}$ & 25.2 \\
500 & 0.013 & 0.799 & 9.8 & 500 & 0.013 & 0.412 & 20.5 \\
500 & 0.032 & 0.252 & 8.1 & 500 & 0.032 & 0.143 & 16.3 \\
500 & 0.080 & $0.627 \times 10^{-1}$ & 9.3 & 500 & 0.080 & $0.368 \times 10^{-1}$ & 18.4 \\
500 & 0.130 & $0.348 \times 10^{-1}$ & 21.4 & 500 & 0.130 & $0.133 \times 10^{-1}$ & 50.5 \\
1000 & 0.013 & 0.482 & 10.2 & 1000 & 0.013 & 0.286 & 19.9 \\
1000 & 0.032 & 0.232 & 6.2 & 1000 & 0.032 & 0.116 & 12.8 \\
1000 & 0.080 & $0.716 \times 10^{-1}$ & 6.4 & 1000 & 0.080 & $0.446 \times 10^{-1}$ & 12.0 \\
1000 & 0.130 & $0.339 \times 10^{-1}$ & 10.9 & 1000 & 0.130 & $0.129 \times 10^{-1}$ & 26.3 \\
2000 & 0.032 & 0.150 & 5.8 & 2000 & 0.032 & $0.717 \times 10^{-1}$ & 12.7 \\
2000 & 0.080 & $0.579 \times 10^{-1}$ & 5.2 & 2000 & 0.080 & $0.234 \times 10^{-1}$ & 12.4 \\
\hline
\end{tabular}


Table 10 continued

Table 11 The $\mathrm{CC} e^{+} p$ cross section $\sigma=d^{2} \sigma^{\mathrm{CC}} / d x d Q^{2}$ for lepton polarisation $P_{e}=-37.0 \%$ (left) and $P_{e}=32.5 \%$ (right) with their statistical $\left(\delta_{\text {stat }}\right)$ uncertainties. The full uncertainties are available in Ref. [58], while the respective cross section values are updated according to Sect. 3 and Ref. [59]

\begin{tabular}{lllccccc}
\hline$Q^{2}\left[\mathrm{GeV}^{2}\right]$ & $x$ & $\sigma\left[\mathrm{pb} / \mathrm{GeV}^{2}\right]$ & $\delta_{\text {stat }}[\%]$ & $Q^{2}\left[\mathrm{GeV}^{2}\right]$ & $x$ & $\sigma\left[\mathrm{pb} / \mathrm{GeV}^{2}\right]$ & $\delta_{\text {stat }}[\%]$ \\
\hline 2000 & 0.130 & $0.293 \times 10^{-1}$ & 7.4 & 2000 & 0.130 & $0.130 \times 10^{-1}$ & 16.3 \\
2000 & 0.250 & $0.106 \times 10^{-1}$ & 14.6 & 2000 & 0.250 & $0.548 \times 10^{-2}$ & 28.9 \\
3000 & 0.080 & $0.402 \times 10^{-1}$ & 5.2 & 3000 & 0.080 & $0.229 \times 10^{-1}$ & 10.1 \\
3000 & 0.130 & $0.236 \times 10^{-1}$ & 6.1 & 3000 & 0.130 & $0.923 \times 10^{-2}$ & 14.3 \\
3000 & 0.250 & $0.865 \times 10^{-2}$ & 9.2 & 3000 & 0.250 & $0.384 \times 10^{-2}$ & 19.6 \\
5000 & 0.080 & $0.265 \times 10^{-1}$ & 6.6 & 5000 & 0.080 & $0.137 \times 10^{-1}$ & 13.6 \\
5000 & 0.130 & $0.158 \times 10^{-1}$ & 5.8 & 5000 & 0.130 & $0.850 \times 10^{-2}$ & 11.7 \\
5000 & 0.250 & $0.609 \times 10^{-2}$ & 7.1 & 5000 & 0.250 & $0.283 \times 10^{-2}$ & 15.1 \\
5000 & 0.400 & $0.185 \times 10^{-2}$ & 19.2 & 5000 & 0.400 & $0.837 \times 10^{-3}$ & 40.9 \\
8000 & 0.130 & $0.107 \times 10^{-1}$ & 7.1 & 8000 & 0.130 & $0.550 \times 10^{-2}$ & 14.3 \\
8000 & 0.250 & $0.325 \times 10^{-2}$ & 7.3 & 8000 & 0.250 & $0.170 \times 10^{-2}$ & 15.4 \\
8000 & 0.400 & $0.132 \times 10^{-2}$ & 13.4 & 8000 & 0.400 & $0.514 \times 10^{-3}$ & 31.7 \\
15,000 & 0.250 & $0.197 \times 10^{-2}$ & 8.4 & 15,000 & 0.250 & $0.103 \times 10^{-2}$ & 17.7 \\
15,000 & 0.400 & $0.492 \times 10^{-3}$ & 11.1 & 15,000 & 0.400 & $0.271 \times 10^{-3}$ & 23.6 \\
30,000 & 0.400 & $0.208 \times 10^{-3}$ & 17.2 & & & & \\
\hline
\end{tabular}

\begin{tabular}{|c|c|c|c|c|c|c|c|}
\hline$Q^{2}\left[\mathrm{GeV}^{2}\right]$ & $x$ & $\sigma\left[\mathrm{pb} / \mathrm{GeV}^{2}\right]$ & $\delta_{\text {stat }}[\%]$ & $Q^{2}\left[\mathrm{GeV}^{2}\right]$ & $x$ & $\sigma\left[\mathrm{pb} / \mathrm{GeV}^{2}\right]$ & $\overline{\delta_{\text {stat }}[\%]}$ \\
\hline 300 & 0.008 & 1.21 & 38.5 & 300 & 0.008 & 0.778 & 49.3 \\
\hline 300 & 0.013 & 0.414 & 28.4 & 300 & 0.013 & 0.593 & 20.4 \\
\hline 300 & 0.032 & 0.102 & 23.6 & 300 & 0.032 & 0.273 & 11.9 \\
\hline 300 & 0.080 & $0.258 \times 10^{-1}$ & 27.5 & 300 & 0.080 & $0.519 \times 10^{-1}$ & 16.8 \\
\hline 500 & 0.013 & 0.286 & 20.4 & 500 & 0.008 & 1.57 & 23.2 \\
\hline 500 & 0.032 & 0.105 & 15.2 & 500 & 0.013 & 0.670 & 11.4 \\
\hline 500 & 0.080 & $0.386 \times 10^{-1}$ & 14.2 & 500 & 0.032 & 0.252 & 8.5 \\
\hline 500 & 0.130 & $0.122 \times 10^{-1}$ & 41.5 & 500 & 0.080 & $0.603 \times 10^{-1}$ & 9.8 \\
\hline 1000 & 0.013 & 0.241 & 18.4 & 500 & 0.130 & $0.268 \times 10^{-1}$ & 23.7 \\
\hline 1000 & 0.032 & 0.124 & 9.9 & 1000 & 0.013 & 0.392 & 12.5 \\
\hline 1000 & 0.080 & $0.204 \times 10^{-1}$ & 13.9 & 1000 & 0.032 & 0.176 & 7.4 \\
\hline 1000 & 0.130 & $0.736 \times 10^{-2}$ & 26.1 & 1000 & 0.080 & $0.512 \times 10^{-1}$ & 7.8 \\
\hline 2000 & 0.032 & $0.537 \times 10^{-1}$ & 11.3 & 1000 & 0.130 & $0.267 \times 10^{-1}$ & 12.1 \\
\hline 2000 & 0.080 & $0.157 \times 10^{-1}$ & 11.5 & 2000 & 0.032 & 0.104 & 7.3 \\
\hline 2000 & 0.130 & $0.698 \times 10^{-2}$ & 17.1 & 2000 & 0.080 & $0.371 \times 10^{-1}$ & 6.6 \\
\hline 2000 & 0.250 & $0.229 \times 10^{-2}$ & 31.8 & 2000 & 0.130 & $0.165 \times 10^{-1}$ & 9.9 \\
\hline 3000 & 0.080 & $0.119 \times 10^{-1}$ & 11.3 & 2000 & 0.250 & $0.473 \times 10^{-2}$ & 19.3 \\
\hline 3000 & 0.130 & $0.544 \times 10^{-2}$ & 14.9 & 3000 & 0.080 & $0.247 \times 10^{-1}$ & 6.9 \\
\hline 3000 & 0.250 & $0.158 \times 10^{-2}$ & 23.1 & 3000 & 0.130 & $0.154 \times 10^{-1}$ & 8.0 \\
\hline 5000 & 0.080 & $0.364 \times 10^{-2}$ & 21.0 & 3000 & 0.250 & $0.260 \times 10^{-2}$ & 15.9 \\
\hline 5000 & 0.130 & $0.309 \times 10^{-2}$ & 15.6 & 5000 & 0.080 & $1.000 \times 10^{-2}$ & 10.9 \\
\hline 5000 & 0.250 & $0.816 \times 10^{-3}$ & 22.5 & 5000 & 0.130 & $0.636 \times 10^{-2}$ & 9.8 \\
\hline 5000 & 0.400 & $0.529 \times 10^{-3}$ & 40.9 & 5000 & 0.250 & $0.187 \times 10^{-2}$ & 13.3 \\
\hline 8000 & 0.130 & $0.696 \times 10^{-3}$ & 29.7 & 5000 & 0.400 & $0.873 \times 10^{-3}$ & 25.0 \\
\hline 8000 & 0.250 & $0.621 \times 10^{-3}$ & 20.5 & 8000 & 0.130 & $0.217 \times 10^{-2}$ & 15.4 \\
\hline 8000 & 0.400 & $0.802 \times 10^{-4}$ & 58.3 & 8000 & 0.250 & $0.100 \times 10^{-2}$ & 13.5 \\
\hline 15,000 & 0.250 & $0.741 \times 10^{-4}$ & 46.0 & 8000 & 0.400 & $0.299 \times 10^{-3}$ & 27.8 \\
\hline \multirow[t]{2}{*}{15,000} & 0.400 & $0.318 \times 10^{-4}$ & 45.0 & 15,000 & 0.250 & $0.315 \times 10^{-3}$ & 19.0 \\
\hline & & & & 15,000 & 0.400 & $0.370 \times 10^{-4}$ & 38.0 \\
\hline
\end{tabular}




\section{Appendix B: Results of fits with many parameters}

Table 12 quotes the fit of $\rho_{\mathrm{NC}}^{\prime}, \kappa_{\mathrm{NC}}^{\prime}$ and $\rho_{\mathrm{CC}}^{\prime}$ parameters and their correlation coefficients. Tables 13, 14, 15, 16, 17 and 18 quote fits of scale-dependent $\rho_{\mathrm{NC}}^{\prime}, \kappa_{\mathrm{NC}}^{\prime}$ and $\rho_{\mathrm{CC}}^{\prime}$ parameters and their correlation coefficients.
Table 12 Results for $\rho_{\mathrm{NC}}^{\prime}, \kappa_{\mathrm{NC}}^{\prime}$ and $\rho_{\mathrm{CC}}^{\prime}$ parameters, and their correlation coefficients, from fits with more than two EW parameters. For the

$\rho_{\mathrm{NC}, d}^{\prime}+\kappa_{\mathrm{NC}, d}^{\prime}+\rho_{\mathrm{NC}, u}^{\prime}+\kappa_{\mathrm{NC}, u}^{\prime}+\mathrm{PDF}$ fit the uncertainties are only approximate since $\chi^{2}$ is not described by a quadratic dependence on the fit parameters. The uncertainties quoted correspond to the total uncertainties

Table 13 Results for the $\rho_{\mathrm{NC}, q}^{\prime}$ and $\kappa_{\mathrm{NC}, q}^{\prime}$ parameters determined at different values of $Q^{2}$. The $Q^{2}$ range of the data selection, and the correlation coefficients of the fit parameters are indicated

\begin{tabular}{|c|c|c|c|c|c|}
\hline Fit parameters & Result & Corre & lation & & \\
\hline \multirow[t]{4}{*}{$\rho_{\mathrm{NC}, u}^{\prime}+\kappa_{\mathrm{NC}, u}^{\prime}+\rho_{\mathrm{NC}, d}^{\prime}+\kappa_{\mathrm{NC}, d}^{\prime}+\mathrm{PDF}$} & $\rho_{\mathrm{NC}, u}^{\prime}=1.53 \pm 0.35$ & 1.00 & & & \\
\hline & $\kappa_{\mathrm{NC}, u}^{\prime}=1.26 \pm 0.14$ & 0.29 & 1.00 & & \\
\hline & $\rho_{\mathrm{NC}, d}^{\prime}=0.18 \pm 0.39$ & -0.86 & -0.26 & 1.00 & \\
\hline & $\kappa_{\mathrm{NC}, d}^{\prime}=-6.4 \pm 10.5$ & -0.84 & -0.34 & 0.993 & 1.00 \\
\hline \multirow[t]{4}{*}{$\rho_{\mathrm{NC}, q}^{\prime}+\kappa_{\mathrm{NC}, q}^{\prime}+\rho_{\mathrm{NC}, e}^{\prime}+\kappa_{\mathrm{NC}, e}^{\prime}+\mathrm{PDF}$} & $\rho_{\mathrm{NC}, q}^{\prime}=1.99 \pm 1.91$ & 1.00 & & & \\
\hline & $\kappa_{\mathrm{NC}, q}^{\prime}=0.93 \pm 0.12$ & -0.02 & 1.00 & & \\
\hline & $\rho_{\mathrm{NC}, e}^{\prime}=0.59 \pm 0.58$ & -0.99 & 0.09 & 1.00 & \\
\hline & $\kappa_{\mathrm{NC}, e}^{\prime}=0.98 \pm 0.06$ & -0.25 & -0.10 & 0.33 & 1.00 \\
\hline \multirow[t]{3}{*}{$\rho_{\mathrm{NC}, f}^{\prime}+\kappa_{\mathrm{NC}, f}^{\prime}+\rho_{\mathrm{CC}, f}^{\prime}+\mathrm{PDF}$} & $\rho_{\mathrm{NC} f}^{\prime}=1.09 \pm 0.07$ & 1.00 & & & \\
\hline & $\kappa_{\mathrm{NC} f}^{\prime}=0.97 \pm 0.05$ & 0.82 & 1.00 & & \\
\hline & $\rho_{\mathrm{CC}, f}^{\prime}=1.004 \pm 0.008$ & 0.03 & -0.12 & 1.00 & \\
\hline
\end{tabular}

\begin{tabular}{llllllllllll}
\hline$Q^{2}$ range $\left[\mathrm{GeV}^{2}\right]$ & Parameter & Result & \multicolumn{1}{l}{ Correlation } & & & & & & \\
\hline$[561,1778]$ & $\rho_{\mathrm{NC}, q}^{\prime}$ & $2.05 \pm 0.50$ & 1.00 & & & & & & \\
{$[1778,6000]$} & $\rho_{\mathrm{NC}, q}^{\prime}$ & $1.06 \pm 0.16$ & 0.11 & 1.00 & & & & & \\
{$[6000,16680]$} & $\rho_{\mathrm{NC}, q}^{\prime}$ & $1.17 \pm 0.18$ & 0.05 & 0.14 & 1.00 & & & & \\
{$[16680,77000]$} & $\rho_{\mathrm{NC}, q}^{\prime}$ & $1.59 \pm 0.42$ & 0.01 & 0.07 & 0.11 & 1.00 & & & & \\
{$[561,1778]$} & $\kappa_{\mathrm{NC}, q}^{\prime}$ & $1.21 \pm 0.15$ & 0.75 & 0.07 & 0.03 & 0.01 & 1.00 & & & \\
{$[1778,6000]$} & $\kappa_{\mathrm{NC}, q}^{\prime}$ & $0.92 \pm 0.16$ & 0.05 & 0.72 & 0.10 & 0.05 & 0.07 & 1.00 & & \\
{$[6000,16680]$} & $\kappa_{\mathrm{NC}, q}^{\prime}$ & $1.02 \pm 0.19$ & 0.02 & 0.09 & 0.63 & 0.07 & 0.03 & 0.09 & 1.00 & \\
{$[16680,77000]$} & $\kappa_{\mathrm{NC}, q}^{\prime}$ & $0.41 \pm 0.33$ & 0.00 & 0.07 & 0.11 & 0.70 & 0.01 & 0.06 & 0.09 & 1.00 \\
\hline
\end{tabular}

Table 14 Results for the $\rho_{\text {NC, } e}^{\prime}$ and $\kappa_{\mathrm{NC}, e}^{\prime}$ parameters determined at different values of $Q^{2}$. The $Q^{2}$ range of the data selection, and the correlation coefficients of the fit parameters are indicated

\begin{tabular}{lllllllllll}
\hline$Q^{2}$ range $\left[\mathrm{GeV}^{2}\right]$ & Parameter & Result & \multicolumn{1}{l}{ Correlation } & & & & & & \\
\hline$[561,1778]$ & $\rho_{\mathrm{NC}, e}^{\prime}$ & $1.51 \pm 0.34$ & 1.00 & & & & & & \\
{$[1778,6000]$} & $\rho_{\mathrm{NC}, e}^{\prime}$ & $1.10 \pm 0.18$ & 0.06 & 1.00 & & & & & \\
{$[6000,16680]$} & $\rho_{\mathrm{NC}, e}^{\prime}$ & $1.14 \pm 0.24$ & 0.03 & 0.17 & 1.00 & & & & & \\
{$[16680,77000]$} & $\rho_{\mathrm{NC}, e}^{\prime}$ & $1.19 \pm 0.34$ & 0.02 & 0.11 & 0.16 & 1.00 & & & & \\
{$[561,1778]$} & $\kappa_{\mathrm{NC}, e}^{\prime}$ & $0.99 \pm 0.06$ & 0.66 & 0.07 & 0.04 & 0.03 & 1.00 & & & \\
{$[1778,6000]$} & $\kappa_{\mathrm{NC}, e}^{\prime}$ & $0.99 \pm 0.07$ & 0.05 & 0.76 & 0.15 & 0.09 & 0.16 & 1.00 & & \\
{$[6000,16680]$} & $\kappa_{\mathrm{NC}, e}^{\prime}$ & $0.98 \pm 0.13$ & 0.02 & 0.15 & 0.83 & 0.13 & 0.07 & 0.16 & 1.00 & \\
{$[16680,77000]$} & $\kappa_{\mathrm{NC}, e}^{\prime}$ & $0.53 \pm 0.21$ & 0.01 & 0.10 & 0.13 & 0.72 & 0.03 & 0.10 & 0.13 & 1.00 \\
\hline
\end{tabular}


Table 15 Results for the $\rho_{\mathrm{NC}, f}^{\prime}$ and $\kappa_{\mathrm{NC}, f}^{\prime}$ parameters determined at different values of $Q^{2}$. The $Q^{2}$ range of the data selection, and the correlation coefficients of the fit parameters are indicated

\begin{tabular}{lllllllllll}
\hline$Q^{2}$ range $\left[\mathrm{GeV}^{2}\right]$ & Parameter & Result & \multicolumn{1}{l}{ Correlation } & & & & & & \\
\hline$[561,1778]$ & $\rho_{\mathrm{NC}, f}^{\prime}$ & $1.28 \pm 0.19$ & 1.00 & & & & & & \\
{$[1778,6000]$} & $\rho_{\mathrm{NC}, f}^{\prime}$ & $1.03 \pm 0.09$ & 0.07 & 1.00 & & & & & \\
{$[6000,16680]$} & $\rho_{\mathrm{NC}, f}^{\prime}$ & $1.07 \pm 0.11$ & 0.03 & 0.19 & 1.00 & & & & & \\
{$[16680,77000]$} & $\rho_{\mathrm{NC}, f}^{\prime}$ & $1.09 \pm 0.17$ & 0.02 & 0.11 & 0.16 & 1.00 & & & & \\
{$[561,1778]$} & $\kappa_{\mathrm{NC}, f}^{\prime}$ & $1.01 \pm 0.07$ & 0.83 & 0.07 & 0.04 & 0.03 & 1.00 & & & \\
{$[1778,6000]$} & $\kappa_{\mathrm{NC}, f}^{\prime}$ & $0.98 \pm 0.06$ & 0.07 & 0.80 & 0.17 & 0.10 & 0.14 & 1.00 & & \\
{$[6000,16680]$} & $\kappa_{\mathrm{NC}, f}^{\prime}$ & $0.99 \pm 0.09$ & 0.03 & 0.16 & 0.83 & 0.13 & 0.06 & 0.18 & 1.00 & \\
{$[16680,77000]$} & $\kappa_{\mathrm{NC}, f}^{\prime}$ & $0.69 \pm 0.13$ & 0.02 & 0.10 & 0.14 & 0.77 & 0.04 & 0.10 & 0.13 & 1.00 \\
\hline
\end{tabular}

Table 16 Results for the $\rho_{\mathrm{CC}, e q}^{\prime}$ parameters determined at different values of $Q^{2}$. The $Q^{2}$ range of the data selection, and the correlation coefficients of the fit parameters are indicated

Table 17 Results for the $\rho_{\mathrm{CC}, e \bar{q}}^{\prime}$ parameters determined at different values of $Q^{2}$. The $Q^{2}$ range of the data selection, and the correlation coefficients of the fit parameters are indicated

Table 18 Results for the $\rho_{\mathrm{CC}, f}^{\prime}$ parameters determined at different values of $Q^{2}$. The $Q^{2}$ range of the data selection, and the correlation coefficients of the fit parameters are indicated

\begin{tabular}{lllllll}
\hline$Q^{2}$ range $\left[\mathrm{GeV}^{2}\right]$ & Parameter & Result & \multicolumn{2}{l}{ Correlation } & \\
\hline$[224,708]$ & $\rho_{\mathrm{CC}, e q}^{\prime}$ & $0.948 \pm 0.030$ & 1.00 & & & \\
{$[708,2239]$} & $\rho_{\mathrm{CC}, e q}^{\prime}$ & $0.993 \pm 0.014$ & 0.40 & 1.00 & & \\
{$[2239,7079]$} & $\rho_{\mathrm{CC}, e q}^{\prime}$ & $0.993 \pm 0.013$ & 0.15 & 0.17 & 1.00 & \\
{$[7079,25119]$} & $\rho_{\mathrm{CC}, e q}^{\prime}$ & $1.008 \pm 0.020$ & -0.03 & 0.01 & 0.12 & 1.00 \\
\hline
\end{tabular}

\begin{tabular}{lllllll}
\hline$Q^{2}$ range $\left[\mathrm{GeV}^{2}\right]$ & Parameter & Result & Correlation & & \\
\hline$[224,708]$ & $\rho_{\mathrm{CC}, e \bar{q}}^{\prime}$ & $1.018 \pm 0.045$ & 1.00 & & & \\
{$[708,2239]$} & $\rho_{\mathrm{CC}, e \bar{q}}^{\prime}$ & $1.054 \pm 0.041$ & 0.63 & 1.00 & & \\
{$[2239,7079]$} & $\rho_{\mathrm{CC}, e \bar{q}}^{\prime}$ & $1.062 \pm 0.046$ & 0.48 & 0.67 & 1.00 & \\
{$[7079,25119]$} & $\rho_{\mathrm{CC}, e \bar{q}}^{\prime}$ & $1.010 \pm 0.075$ & 0.14 & 0.29 & 0.50 & 1.00 \\
\hline
\end{tabular}

\begin{tabular}{lllllll}
\hline$Q^{2}$ range $\left[\mathrm{GeV}^{2}\right]$ & Parameter & Result & \multicolumn{2}{l}{ Correlation } & & \\
\hline$[224,708]$ & $\rho_{\mathrm{CC}, f}^{\prime}$ & $0.976 \pm 0.018$ & 1.00 & & & \\
{$[708,2239]$} & $\rho_{\mathrm{CC}, f}^{\prime}$ & $0.998 \pm 0.011$ & 0.47 & 1.00 & & \\
{$[2239,7079]$} & $\rho_{\mathrm{CC}, f}^{\prime}$ & $0.999 \pm 0.010$ & 0.19 & 0.24 & 1.00 & \\
{$[7079,25119]$} & $\rho_{\mathrm{CC}, f}^{\prime}$ & $1.004 \pm 0.017$ & -0.06 & -0.01 & 0.12 & 1.00 \\
\hline
\end{tabular}

\section{References}

1. Gargamelle Neutrino Collaboration, F.J. Hasert et al. Phys. Lett. 46B, 138 (1973)

2. D. Haidt, Adv. Ser. Direct. High Energy Phys. 23, 165 (2015)

3. S.L. Glashow, Nucl. Phys. 22, 579 (1961)

4. S. Weinberg, Phys. Rev. Lett. 19, 1264 (1967)

5. S. Weinberg, Phys. Rev. Lett. 27, 1688 (1971)

6. S. Weinberg, Phys. Rev. D 5, 1412 (1972)

7. A. Salam, J.C. Ward, Phys. Lett. 13, 168 (1964)

8. P.W. Higgs, Phys. Lett. 12, 132 (1964)

9. P.W. Higgs, Phys. Rev. Lett. 13, 508 (1964)

10. F. Englert, R. Brout, Phys. Rev. Lett. 13, 321 (1964)

11. C.Y. Prescott et al., Phys. Lett. 77B, 347 (1978)

12. C.Y. Prescott et al., Phys. Lett. 84B, 524 (1979)

13. V. MuLan Collaboration, Tishchenko et al. Phys. Rev. D 87, 052003 (2013). arXiv: 1211.0960

14. G.L. Fogli, D. Haidt, Z. Phys. C 40, 379 (1988)

15. A. Blondel et al., Z. Phys. C 45, 361 (1990)
16. CHARM Collaboration, J.V. Allaby et al., Z. Phys. C 36, 611 (1987)

17. E770, E744, CCFR Collaboration, K.S. McFarland et al., Eur. Phys. J. C 1, 509 (1998). arXiv:hep-ex/9701010

18. NuTeV Collaboration, G.P. Zeller et al., Phys. Rev. Lett. 88, 091802 (2002). arXiv:hep-ex/0110059 [Erratum: Phys. Rev. Lett. 90, 239902 (2003)]

19. SLD Electroweak Group, DELPHI, ALEPH, SLD, SLD Heavy Flavour Group, OPAL, LEP Electroweak Working Group, L3 Collaboration, S. Schael et al., Phys. Rep. 427, 257 (2006). arXiv:hep-ex/0509008

20. CMS Collaboration, S. Chatrchyan et al., Phys. Rev. D 84, 112002 (2011). arXiv: 1110.2682

21. DELPHI, OPAL, LEP Electroweak, ALEPH, L3 Collaboration, S. Schael et al., Phys. Rep. 532, 119 (2013). arXiv:1302.3415

22. LHCb Collaboration, R. Aaij et al., JHEP 11, 190 (2015). arXiv: 1509.07645

23. ATLAS Collaboration, G. Aad et al., JHEP 09, 049 (2015). arXiv:1503.03709 
24. CDF, D0 Collaboration, T.A. Aaltonen et al., Phys. Rev. D 97, 112007 (2018). arXiv:1801.06283

25. H1 Collaboration, T. Ahmed et al., Phys. Lett. B 324, 241 (1994)

26. H1 Calorimeter Group Collaboration, B. Andrieu et al., Nucl. Instrum. Methods A 336, 460 (1993)

27. H1 Collaboration, I. Abt et al., Nucl. Instrum. Methods A 386, 310 (1997)

28. H1 Collaboration, I. Abt et al., Nucl. Instrum. Methods A 386, 348 (1997)

29. H1 SPACAL Group Collaboration, R.D. Appuhn et al., Nucl. Instrum. Methods A 386, 397 (1997)

30. H1 Collaboration, S. Aid et al., Phys. Lett. B 379, 319 (1996). arXiv:hep-ex/9603009

31. ZEUS Collaboration, J. Breitweg et al., Eur. Phys. J. C 12, 411 (2000). arXiv:hep-ex/9907010 [Erratum: Eur. Phys. J. C 2, 305 (2003)]

32. H1 Collaboration, C. Adloff et al., Eur. Phys. J. C 13, 609 (2000). arXiv:hep-ex/9908059

33. H1 Collaboration, C. Adloff et al., Eur. Phys. J. C 19, 269 (2001). arXiv:hep-ex/0012052

34. ZEUS Collaboration, S. Chekanov et al., Phys. Lett. B 539, 197 (2002). arXiv:hep-ex/0205091 [Erratum: Phys. Lett. B 552, 308 (2003)]

35. H1 Collaboration, A. Aktas et al., Phys. Lett. B 632, 35 (2006). arXiv:hep-ex/0507080

36. ZEUS Collaboration, H. Abramowicz et al., Phys. Rev. D 93, 092002 (2016). arXiv: 1603.09628

37. I. Abt et al., Phys. Rev. D 94, 052007 (2016). arXiv:1604.05083

38. A.M. Cooper-Sarkar, A. Bodek, K. Long, E. Rizvi, H. Spiesberger, J. Phys. G 25, 1387 (1999). arXiv:hep-ph/9902277

39. M. Klein, T. Riemann, Z. Phys. C 24, 151 (1984)

40. Particle Data Group Collaboration, C. Patrignani et al., Chin. Phys. C 40, 100001 (2016)

41. A. Sirlin, Phys. Rev. D 22, 971 (1980)

42. A. Sirlin, Phys. Rev. D 29, 89 (1984)

43. M. Böhm, H. Spiesberger, W. Hollik, Fortsch. Phys. 34, 687 (1986)

44. W.F.L. Hollik, Fortsch. Phys. 38, 165 (1990)

45. M. Böhm, H. Spiesberger, Nucl. Phys. B 294, 1081 (1987)

46. D.Yu. Bardin, C. Burdik, P.C. Khristova, T. Riemann, Z. Phys. C 42, 679 (1989)

47. W. Hollik, D.Yu. Bardin, J. Blümlein, B.A. Kniehl, T. Riemann, H. Spiesberger, Electroweak parameters at HERA: theoretical aspects, in Workshop on Physics at HERA Hamburg, Germany, October 29-30, 1991 (1992), p. 923 (MPI-PH-92-30)

48. M. Böhm, H. Spiesberger, Nucl. Phys. B 304, 749 (1988)

49. D.Yu. Bardin, K.C. Burdik, P.K. Khristova, T. Riemann, Z. Phys. C 44, 149 (1989)

50. B. Heinemann, S. Riess, H. Spiesberger, Radiative corrections for charged current scattering: a comparison of computer codes, in Monte Carlo Generators for HERA Physics. Proceedings, Workshop, Hamburg, Germany 1998-1999 (1998), p. 530

51. H. Spiesberger, EPRC: a program package for electroweak physics at HERA, in Future Physics at HERA. Proceedings, Workshop, Hamburg, Germany, September 25, 1995-May 31, 1996, vols. 1, 2 (1995)

52. A. Kwiatkowski, H. Spiesberger, H.J. Möhring, Comput. Phys. Commun. 69, 155 (1992)

53. K. Charchula, G.A. Schuler, H. Spiesberger, Comput. Phys. Commun. 81, 381 (1994)

54. H. Spiesberger, Adv. Ser. Direct. High Energy Phys. 14, 626 (1995)

55. W.J. Marciano, A. Sirlin, Phys. Rev. D 22, 2695 (1980) [Erratum: Phys. Rev. D 31, 213 (1985)]

56. H1 Collaboration, F.D. Aaron et al., Eur. Phys. J. C 71, 1579 (2011). arXiv:1012.4355
57. H1 Collaboration, C. Adloff et al., Eur. Phys. J. C 30, 1 (2003). arXiv:hep-ex/0304003

58. H1 Collaboration, F.D. Aaron et al., JHEP 09, 061 (2012). arXiv: 1206.7007

59. H1 Collaboration, F.D. Aaron et al., Eur. Phys. J. C 72, 2163 (2012). arXiv:1205.2448 [Erratum: Eur. Phys. J. C 74, 2733 (2012)]

60. H1 Collaboration, C. Adloff et al., Nucl. Phys. B 497, 3 (1997). arXiv:hep-ex/9703012

61. H1 Collaboration, C. Adloff et al., Eur. Phys. J. C 21, 33 (2001). arXiv:hep-ex/0012053

62. H1 Collaboration, F.D. Aaron et al., Eur. Phys. J. C 63, 625 (2009). arXiv:0904.0929

63. H1 Collaboration, F.D. Aaron et al., Eur. Phys. J. C 64, 561 (2009). arXiv:0904.3513

64. A. Vogt, S. Moch, J.A.M. Vermaseren, Nucl. Phys. B 691, 129 (2004). arXiv:hep-ph/0404111

65. S. Moch, J.A.M. Vermaseren, A. Vogt, Nucl. Phys. B 688, 101 (2004). arXiv:hep-ph/0403192

66. H1 and ZEUS Collaborations, H. Abramowicz et al., Eur. Phys. J. C 75, 580 (2015). arXiv: 1506.06042

67. H1 Collaboration, V. Andreev et al., Eur. Phys. J. C 77, 791 (2017). arXiv: 1709.07251

68. S. Alekhin et al., Eur. Phys. J. C 75, 304 (2015). arXiv:1410.4412

69. M. Botje, Comput. Phys. Commun. 182, 490 (2011). arXiv:1005.1481

70. M. Botje, arXiv: 1602.08383

71. H1 Collaboration, V. Andreev et al., Eur. Phys. J. C 75, 65 (2015). arXiv: 1406.4709

72. B. Sobloher, R. Fabbri, T. Behnke, J. Olsson, D. Pitzl, S. Schmitt, J. Tomaszewska, arXiv:1201.2894

73. J. Blümlein, M. Klein, T. Riemann, Testing the electroweak Standard Model at HERA, in Physics at Future Accelerators, Proceedings, 10th Warsaw Symposium on Elementary Particle Physics, Kazimierz, Poland, May 24-30, 1987 (1987), p. 39 (PHE-87-03)

74. G. Cowan, K. Cranmer, E. Gross, O. Vitells, Eur. Phys. J. C 71, 1554 (2011). arXiv:1007.1727 [Erratum: Eur. Phys. J. C 73, 2501 (2013)]

75. F. James, M. Roos, Comput. Phys. Commun. 10, 343 (1975)

76. ALEPH Collaboration, R. Barate et al., Phys. Lett. B 415, 435 (1997)

77. L3 Collaboration, M. Acciarri et al., Phys. Lett. B 407, 419 (1997)

78. OPAL Collaboration, K. Ackerstaff et al., Phys. Lett. B 389, 416 (1996)

79. DELPHI Collaboration, J. Abdallah et al., Eur. Phys. J. C 55, 1 (2008). arXiv:0803.2534

80. D0 Collaboration, V.M. Abazov et al., Phys. Rev. Lett. 108, 151804 (2012). arXiv:1203.0293

81. CDF Collaboration, T. Aaltonen et al., Phys. Rev. Lett. 108, 151803 (2012). arXiv: 1203.0275

82. CDF Collaboration, T.A. Aaltonen et al., Phys. Rev. D 89, 072003 (2014). arXiv:1311.0894

83. ATLAS Collaboration, M. Aaboud et al., Eur. Phys. J. C 78, 110 (2018). arXiv: 1701.07240

84. CDF, D0 Collaboration, T.A. Aaltonen et al., Phys. Rev. D 88, 052018 (2013). arXiv:1307.7627

85. R. Beyer, E. Elsen, S. Riess, F. Zetsche, H. Spiesberger, Electroweak precision tests with deep inelastic scattering at HERA, in Future Physics at HERA. Proceedings, Workshop, Hamburg, Germany, September 25, 1995-May 31, 1996, vols. 1, 2 (1995)

86. V. Brisson, F.W. Büsser, F. Niebergall, E. Elsen, D. Haidt, M. Hapke, M. Kuhlen, The Measurement of electroweak parameters at HERA, in Workshop on Physics at HERA Hamburg, Germany, October 29-30, 1991 (1991), p. 947

87. ATLAS Collaboration, G. Aad et al., Eur. Phys. J. C 75, 330 (2015). arXiv: 1503.05427 
88. CMS Collaboration, V. Khachatryan et al., Phys. Rev. D 93, 072004 (2016). arXiv:1509.04044

89. CMS Collaboration, A.M. Sirunyan et al., Phys. Rev. D 96, 032002 (2017). arXiv:1704.06142

90. ATLAS Collaboration, M. Aaboud et al., JHEP 09, 118 (2017). arXiv: 1702.07546

91. CMS Collaboration, Measurement of the top quark mass with lepton+jets final states in $p p$ collisions at $\sqrt{s}=13 \mathrm{TeV}$. CMS-PASTOP-17-007, CERN, Geneva (2017). https://cds.cern.ch/record/ 2284594

92. CDF and D0 Collaboration, T.E.W. Group, T. Aaltonen, arXiv: 1608.01881

93. ATLAS, CMS Collaboration, G. Aad et al., Phys. Rev. Lett. 114, 191803 (2015). arXiv:1503.07589

94. J. de Blas, M. Ciuchini, E. Franco, S. Mishima, M. Pierini, L. Reina, L. Silvestrini, JHEP 12, 135 (2016). arXiv:1608.01509

95. J. Haller, A. Hoecker, R. Kogler, K. Mönig, T. Peiffer, J. Stelzer, Eur. Phys. J. C 78, 675 (2018). arXiv:1803.01853
96. D0 Collaboration, V.M. Abazov et al., Phys. Rev. D 84, 012007 (2011). arXiv:1104.4590

97. S. Alekhin, J. Blümlein, S. Moch, R. Placakyte, Phys. Rev. D 96, 014011 (2017). arXiv:1701.05838

98. S. Dulat, T.-J. Hou, J. Gao, M. Guzzi, J. Huston, P. Nadolsky, J. Pumplin, C. Schmidt, D. Stump, C.P. Yuan, Phys. Rev. D 93, 033006 (2016). arXiv:1506.07443

99. L.A. Harland-Lang, A.D. Martin, P. Motylinski, R.S. Thorne, Eur. Phys. J. C 75, 204 (2015). arXiv:1412.3989

100. NNPDF Collaboration, R.D. Ball et al., JHEP 04, 040 (2015). arXiv: 1410.8849

101. H1 Collaboration, F.D. Aaron et al., Phys. Lett. B 704, 388-396 (2011). arXiv:1107.3716

102. H1 Collaboration, F.D. Aaron et al., Phys. Lett. B 705, 52-58 (2011). arXiv:1107.2478 